\title{
CHY-construction of planar loop integrands of cubic scalar theory
}

\section{Bo Feng}

Zhejiang Institute of Modern Physics, Physics Department, Zhejiang University, Hangzhou, Zhejiang, 310027, P.R. China

Niels Bohr International Academy and Discovery Center, The Niels Bohr Institute, University of Copenhagen,

Blegdamsvej 17, DK-2100 Copenhagen, Denmark

E-mail: b.feng@cms.zju.edu.cn

ABSTRACT: In this paper, by treating massive loop momenta as massless momenta in higher dimensions, we are able to treat all-loop scattering equations as tree ones. As an application of the new perspective, we consider the CHY-construction of bi-adjoint $\phi^{3}$ theory. We present the explicit formula for two-loop planar integrands. We discuss in details how to subtract various forward singularities in the construction. We count the number of terms obtained by our formula and by direct Feynman diagram calculation and find the perfect match, thus provide a strong support for our results.

Keywords: Scattering Amplitudes, Field Theories in Higher Dimensions

ARXIV EPRINT: 1601.05864 


\section{Contents}

1 Introduction 1

2 Tree-level amplitude of color-ordered bi-adjoint $\phi^{3}$ theory 3

$\begin{array}{llr}3 & \text { All-Loop scattering equations } & 8\end{array}$

4 Two-loop CHY-integrand of $\phi^{3}$ theory 12

4.1 Analysis of two-loop Feynman diagrams 12

$\begin{array}{ll}4.2 \text { Special Feynman diagrams } & 16\end{array}$

$\begin{array}{lll}4.3 & \text { The construction of CHY-integrand } & 19\end{array}$

4.3.1 The CHY-integrand for ordering $\mathcal{O}_{j k} \quad 20$

4.3.2 The CHY-integrand for ordering $\mathcal{O}_{j} \quad 24$

4.4 The CHY-construction of reducible two-loop diagrams 25

5 Counting $\quad 28$

5.1 Counting from CHY-formula 28

5.2 Counting from Feynman diagrams 30

6 Conclusion $\quad 31$

\section{Introduction}

The discovery of CHY-formula for tree-level scattering amplitudes by Cachazo, He and Yuan $[\mathrm{CHY}]$ in a series of papers [1-5] has provided a novel way to calculate and understand scattering amplitudes. In this construction, a set of algebraic equations ( called the scattering equations) has played a crucial role. These equations appear in the literature in a variety of contexts [6-14]. More explicitly, scattering equations of $n$-particles are given by

$$
\mathcal{S}_{a} \equiv \sum_{b \neq a} \frac{2 k_{a} \cdot k_{b}}{z_{a b}}=0, \quad z_{a b} \equiv z_{a}-z_{b}, \quad a=1,2, \ldots, n
$$

where the $z_{a}$ is the location of $a$-th particle on the Riemann surface. Although there are $n$ equations, only $(n-3)$ of them are independent, which can be seen by following three identities:

$$
\sum_{a} \mathcal{S}_{a}=0, \quad \sum_{a} \mathcal{S}_{a} z_{a}=0, \quad \sum_{a} \mathcal{S}_{a} z_{a}^{2}=0
$$


under the momentum conservation and null conditions $k_{a}^{2}=0$. The tree-level amplitude is calculated by the following formula

$$
\mathcal{A}_{n}=\int \frac{\left(\prod_{a=1}^{n} d z_{a}\right)}{d \omega} \Omega(\mathcal{S}) \mathcal{I}, \quad d \omega=\frac{d z_{r} d z_{s} d z_{t}}{z_{r s} z_{s t} z_{t r}}
$$

where $\mathcal{I}$ is the so called $C H Y$-integrand and $d \omega$ is the volume of the $\operatorname{SL}(2, C)$ gauge group, where we have used this symmetry to fix three variables $z_{r}, z_{s}, z_{t}$. The $\Omega(\mathcal{S})$ is given by

$$
\Omega(\mathcal{S})_{i j m}=z_{i j} z_{j m} z_{m i} \prod_{a \neq i, j, k, m} \delta\left(\mathcal{S}_{a}\right)
$$

where $(n-3)$-independent delta-functions of scattering equations have been imposed. Since there are $(n-3)$ variables and $(n-3)$ equations, there is no integration to be done in (1.3). For each solution of these equations, we get a contribution after inserting it into the CHYintegrand $\mathcal{I}$. The amplitude is obtained by summing all $(n-3)$ ! contributions.

The CHY-formula has been confirmed from various perspectives. In [15], using the $\mathrm{BCFW}$ on-shell recursion relation $[16,17]$ the validity of the CHY construction for $\phi^{3}$ theory and Yang-Mills theory has been proved. Using ambitwistor string theory [18-28], by calculating corresponding correlation functions of different world-sheet fields, different CHY-formulas for different theories have been derived together with the natural appearance of scattering equations. In [29], inspired by the field theory limit of string theory, a dual model has been introduced. Using this idea, a direct connection between the CHY-formula and the standard tree-level Feynman diagrams has been established in [30, 31].

The CHY-formula (or CHY-construction) has seperated calculating scattering amplitudes of a given theory into two parts: (a) Finding solutions of scattering equations and (b) Figuring out the corresponding CHY-integrand $\mathcal{I}$, which is a rational function of locations $z_{a}$ for the given theory. Between these two parts, the former task is universal for all theories while the latter does depend on the detail of a particular theory. Although there are some general guiding principles for the construction of CHY-integrands, we still do not know the general algorithm for all theories. However, amazing progress has been made in [5] where integrands are known for many theories.

Although looking simple, scattering equations are not easy to solve. By some proper transformations, scattering equations become a set of polynomial equations as shown in [33]. Based on this technique, several works have been done [34-39] by exploring the powerful computational algebraic geometry method, such as the companion matrix, the Bezoutian matrix and the elimination theorem. A different approach is given in [40] by mapping the problem to the known result of bi-adjoint $\phi^{3}$ theory. Using the generalized KLT relation and Hamiltonian decompositions of certain 4-regular graphs, one can bypass solving scattering equations and read out results directly. Another powerful method is given in $[30,31]$, where a mapping rule between CHY-integrands and tree-level Feynman diagrams has been provided. In this paper, we will use the mapping rule extensively.

Encouraged by the success at tree-level, a lot of effort have been done to generalize to loop-level [21, 24, 25]. A breakthrough is given in [28] by Geyer, Mason, Monteiro and Tourkine [28]. They show how to reduce the problem of one-loop on genus one surface to 
a modified problem on the Riemann sphere, thus the one-loop analysis is essentially as the tree-level one. Using this picture, they provide a conjecture to any loop order. In [32, 41], the one-loop integrand of bi-adjoint $\phi^{3}$ theory has been proposed, while in $[42,43]$ more general theories such as Yang-Mills theory and gravity have been treated at the one-loop level. Among these results, the generalization of mapping rule to one-loop level given in [32] will be very useful. In fact, in this paper, we will show that this mapping rule could be generalized to all loops.

In this paper, we will generalize the above one-loop results to higher loops. We will write down all-loop scattering equations. The key idea of our approach is to treat massive loop momenta as massless momenta in a higher dimensions. Using the idea, we effectively reduce the loop problem to the tree one. In fact, the same idea has been explored by the $\mathcal{Q}$ cut construction in $[44,45]$. After having loop-level scattering equations, we construct the CHY-integrand, which will produce the two-loop planar integrand of bi-adjoint $\phi^{3}$ theory.

The plan of the paper is as follows. In section 2, we have reviewed the mapping rule between CHY-integrands and Feynman diagrams of bi-adjoint $\phi^{3}$ theory and discussed how to write down CHY-integrands for tree diagrams with a given set of poles. In section 3, we discuss all-loop scattering equations. In section 4, we construct the two-loop CHYintegrand for $\phi^{3}$ theory. To carry out the construction, we have carefully discussed the related forward singularities when sewing trees into loops and how to remove them. In section 5, by matching the number of terms obtained by CHY-construction and by Feynman diagrams, we provide a strong support for our result. In section 6 , a brief conclusion is given.

\section{Tree-level amplitude of color-ordered bi-adjoint $\phi^{3}$ theory}

In this section, we will review relevant results of color ordered bi-adjoint $\phi^{3}$ theory at tree-level, especially the mapping rule between tree-level Feynman diagrams and tree-level CHY-integrands. Using this mapping rule, we can discuss how to remove certain Feynman diagrams from a given CHY-integrand. Before doing so, let us define the following compact notation

$$
\left[i_{1}, i_{2}, \ldots, i_{m}\right] \equiv \sum_{1 \leq a<b \leq m} 2 k_{i_{a}} \cdot k_{i_{b}}
$$

Now we discuss the mapping rule given in $[30,31]$. First it is worth noticing that by Mobius invariance each factor $z_{i}$ should have degree -4 in the CHY-integrand, thus one can represent the CHY-integrand by a graph, where each factor $z_{i j} \equiv\left(z_{i}-z_{j}\right)$ in the denominator corresponds to one (arrowed) solid line connecting vertices $i, j$ and each factor $z_{i j}$ in the numerator corresponds to one (arrowed) dash line connecting vertices $i, j$. Such a graph will be called the CHY-graph. Given a CHY-integrand (or CHY-graph), the result obtained from CHY-formula would be a sum of inverse-products of multi-index Mandelstam invariants denoted $s_{i_{1} \ldots i_{m}}=\left(k_{i_{1}}+\ldots+k_{i_{m}}\right)^{2}=\left[i_{1}, i_{2}, \ldots, i_{m}\right]$ for which $k_{i}^{2}=0$, i.e.,

$$
\prod_{a=1}^{n-3} 1 / s_{P_{a}}=\prod_{a=1}^{n-3} \frac{1}{\left[P_{a}\right]}
$$




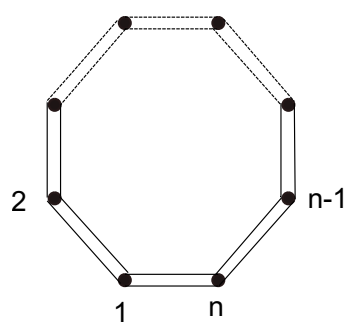

(a)

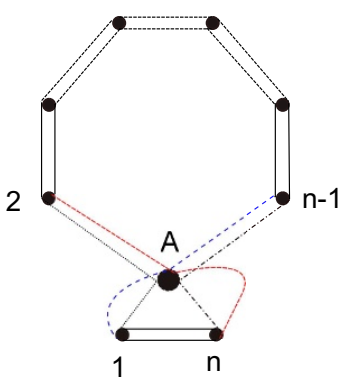

(b)

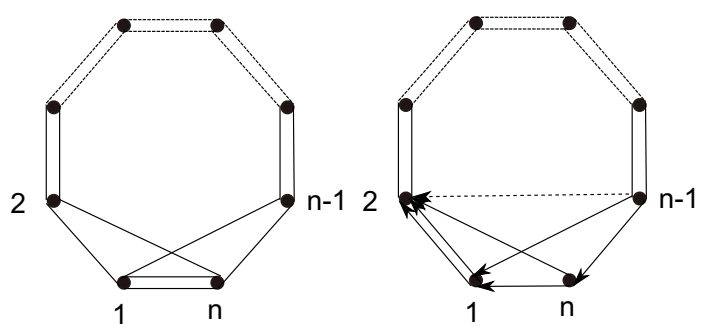

(c)

(d)

Figure 1. The CHY-graph for Feynman diagrams with pole $s_{n 1}$. (a) The CHY-graph for full $n$-point tree-level amplitude; (b) The pinching picture where the new vertex $A$ represents the combination of vertices $1, n$. (c) The CHY-graph for Feynman diagrams containing pole $s_{n 1}$ obtained from (b) after lifting the $(n-1)$-point graph to the $n$-point graph. (d) The CHY-graph after subtracting (c) from (a), where we have used arrows to indicate the direction.

for $n$-point tree-level amplitudes. Each $P_{a} \subset\{1, \ldots, n\}$ denotes a subset of legs that includes at most $n / 2$ elements (because $s_{P}=s_{P}$, with $P^{\complement} \equiv \mathbb{Z}_{n} \backslash P$, by momentum conservation). The collections of subsets $\left\{P_{a}\right\}$ appearing in (2.2) must satisfy the following criteria:

- for each pair of indices $\{i, j\} \subset P_{a}$ in each subset $P_{a}$, there are exactly $\left(2\left|P_{a}\right|-2\right)$ factors of $\left(z_{i}-z_{j}\right)$ appearing in the denominator of $\mathcal{I}\left(z_{1}, \ldots, z_{n}\right)$;

- each pair of subsets $\left\{P_{a}, P_{b}\right\}$ in the collection is either nested or complementary that is, $P_{a} \subset P_{b}$ or $P_{b} \subset P_{a}$ or $P_{a} \subset P_{b}^{\complement}$ or $P_{b}^{\complement} \subset P_{a}$;

If there are no collections of $(n-3)$ subsets $\left\{P_{a}\right\}$ satisfying the criteria above, the result of integration will be zero. One simple example using this rule is

$$
\frac{1}{z_{12}^{2} z_{23} z_{34} z_{45} z_{15} z_{35}^{2} z_{14} z_{24}} \Longleftrightarrow \frac{1}{s_{12} s_{35}}
$$

Another important example is the CHY-integrand for the full tree-level amplitude of $\phi^{3}$ theory with ordering $\{1,2, \ldots, n\}$ (the corresponding CHY-graph is given by diagram (a) in figure 1)

$$
\mathcal{I}_{n}^{\mathrm{CHY}}(\{1,2, \ldots, n\})=\frac{1}{z_{12}^{2} z_{23}^{2} \ldots z_{(n-1) n}^{2} z_{n 1}^{2}} .
$$

There is one fundamental formula, which will be useful later: the number of color-ordered $n$-point tree-level Feynman diagrams of $\phi^{3}$ theory is

$$
C(n)=\frac{2^{n-2}(2 n-5) ! !}{(n-1) !}
$$

Having presented the rule above, we try to find the CHY-integrand which gives Feynman diagrams of certain type, such as those in figure 3 and figure 8. Let us start with the simplest case, i.e., the (B-2) type of figure 3, where we assume that $1, n$ are always attached to the same cubic vertex and then they merge together to be connected to other 


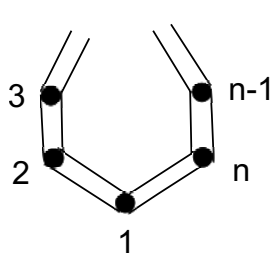

(a)

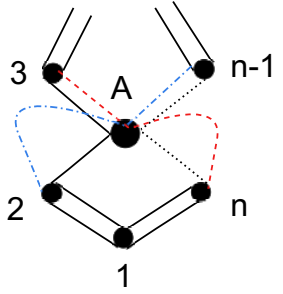

(b)

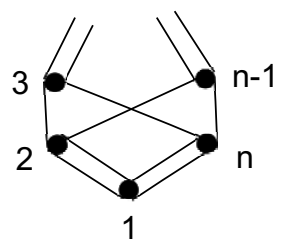

(c)

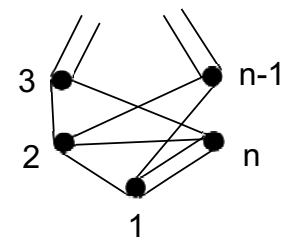

(d)

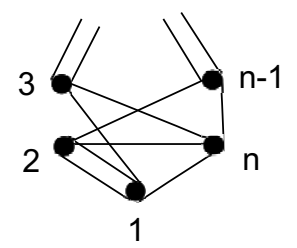

(e)

Figure 2. (a) The CHY-graph for $n$-point tree-level amplitude; (b) The pinching picture where the new vertex $A$ represents the combination of vertices $n, 1,2$. (c) The CHY-graph for all Feynman diagrams with pole $s_{n 12}$ obtained from (b) after lifting the $(n-2)$-point graph to the $n$-point graph. (d) The CHY-graph containing the fixed poles $s_{n 12}$ and $s_{n 1}$; (e) The CHY-graph containing the fixed poles $s_{n 12}$ and $s_{12}$.

legs. If we cut the propagator $s_{1 n}$, we will be left with color ordered full tree-level amplitude with $(n-1)$-legs. This picture motivates us to use an operation called the pinching where vertices $1, n$ merge and become a new vertex $A$ (see diagram (b) in figure 1 ). It is worth noticing that in (b) we have drawn four lines in different colors and styles to emphasize when we lift the $(n-1)$-point graph to $n$-point graph, how these lines are connected. Also the group $A, 1,2$ itself is the CHY-graph corresponding to the expression (2.4) with $n=3$. The lifted graph of (b) is given in diagram (c) in figure 1. When translating the above graphic manipulation to an expression, we find that

$$
\mathcal{I}_{n ; s_{n 1}}^{\mathrm{CHY}}(\{1,2, \ldots, n\})=\frac{1}{z_{23}^{2} \ldots z_{(n-2)(n-1)}^{2}\left(z_{(n-1) n} z_{(n-1) 1}\left(z_{n 1}^{2}\right) z_{n 2} z_{12}\right)}
$$

Using the mapping rule, one can check that the CHY-integrand (2.6) will give an expression containing $C(n-1)$ terms with the fixed pole $s_{n 1}$ (see eq.(2.5)), which is the correct number. Now it is obvious that if we want to remove these Feynman diagrams of the (B-2) type, we should subtract the CHY-integrand (2.6) from (2.4) and get

$$
\begin{aligned}
& \frac{1}{z_{23}^{2} \ldots z_{(n-2)(n-1)}^{2}}\left\{\frac{1}{z_{(n-1) n}^{2} z_{n 1}^{2} z_{12}^{2}}-\frac{1}{z_{(n-1) n} z_{(n-1) 1} z_{n 1}^{2} z_{n 2} z_{12}}\right\} \\
& =\frac{z_{(n-1) 2}}{z_{23}^{2} \ldots z_{(n-2)(n-1)}^{2}} \frac{z_{(n-1) n}^{2} z_{(n-1) 1} z_{n 1} z_{n 2} z_{12}^{2}}{z^{2}}
\end{aligned}
$$

where the explicit pole $z_{n 1}^{2}$ in the denominator has been canceled. The final CHY-integrand can be represented by diagram (d) in figure 1 .

Having done the simplest case, now we consider the CHY-integrand, which produces all Feynman diagrams containing a given pole, for example, $s_{n 12}$. Again we can pinch three vertices $n, 1,2$ together to reduce $n$-legs to $(n-2)$-legs (see diagram (b) in figure 2 ). After lifting we get the CHY-graph (see diagram (c) in figure 2), which contains all Feynman diagrams containing the pole $s_{n 12}$. The corresponding CHY-integrand is obtained by replacing $z_{(n-1) n}^{2}\left(z_{n 1}^{2} z_{12}^{2}\right) z_{23}^{2}$ in the denominator of $(2.4)$ by $z_{(n-1) n} z_{(n-1) 2}\left(z_{n 1}^{2} z_{12}^{2}\right) z_{n 3} z_{23}$, i.e.,

$$
\mathcal{I}_{n ; s_{n 12}}^{\mathrm{CHY}}(\{1,2, \ldots, n\})=\frac{1}{z_{34}^{2} \ldots z_{(n-2)(n-1)^{2}}\left(z_{(n-1) n} z_{(n-1) 2}\left(z_{n 1}^{2} z_{12}^{2}\right) z_{n 3} z_{23}\right)}
$$


If we subtract the CHY-integrand (2.8) from (2.4), we will get

$$
\begin{aligned}
& \frac{1}{z_{34}^{2} \ldots z_{(n-2)(n-1)}^{2}}\left\{\frac{1}{z_{(n-1) n}^{2}\left(z_{n 1}^{2} z_{12}^{2}\right) z_{23}^{2}}-\frac{1}{z_{(n-1) n} z_{(n-1) 2}\left(z_{n 1}^{2} z_{12}^{2}\right) z_{n 3} z_{23}}\right\} \\
& =\frac{z_{(n-1) 3} z_{n 2}}{z_{34}^{2} \ldots z_{(n-2)(n-1)}^{2}} \frac{z_{(n-1) n}^{2} z_{(n-1) 2}\left(z_{n 1}^{2} z_{12}^{2}\right) z_{n 3} z_{23}^{2}}{} .
\end{aligned}
$$

Using the pinching operation this pattern can be easily generalized to find the CHYintegrand which produces all Feynman diagrams containing a given pole, for example, $s_{n 12 \ldots k}$. What we need to do is the following replacement of $z_{(n-1) n}^{2}\left(z_{n 1}^{2} z_{12}^{2} \ldots z_{(k-1) k}^{2}\right) z_{k(k+1)}^{2}$ in the denominator of $(2.4)$ by $z_{(n-1) n} z_{(n-1) k}\left(z_{n 1}^{2} z_{12}^{2} \ldots z_{(k-1) k}^{2}\right) z_{n(k+1)} z_{k(k+1)}$, i.e.,

$$
\begin{aligned}
& \frac{1}{\ldots z_{(n-1) n}^{2}\left(z_{n 1}^{2} z_{12}^{2} \ldots z_{(k-1) k}^{2}\right) z_{k(k+1)}^{2} \cdots} \Longrightarrow \\
& \frac{1}{\ldots z_{(n-1) n} z_{(n-1) k}\left(z_{n 1}^{2} z_{12}^{2} \ldots z_{(k-1) k}^{2}\right) z_{n(k+1)} z_{k(k+1)} \cdots} .
\end{aligned}
$$

The above replacement rule can be nicely represented as the following: for each fixed pole $s_{n 12 \ldots k}$ we multiply it by a corresponding factor

$$
\mathcal{P}[n-1, n, k, k+1] \equiv \frac{z_{(n-1) n} z_{k(k+1)}}{z_{(n-1) k} z_{n(k+1)}}
$$

where $n, k$ are the first and the last legs in the ordering of the specific pole.

Having observed the pattern, now it is easy to write down the corresponding CHYintegrand with a given pole structure (we will call it as the signature). Let us give a few examples:

- With fixed poles $s_{n 12}$ and $s_{456}$, the integrand is given by $\mathcal{I}_{n}^{\mathrm{CHY}}(\{1,2, \ldots, n\}) \mathcal{P}[n-$ $1, n, 2,3] \mathcal{P}[3,4,6,7]$.

- With fixed poles $s_{12}$ and $s_{34}$, the integrand is given by $\mathcal{I}_{n}^{\mathrm{CHY}}(\{1,2, \ldots, n\}) \mathcal{P}[n, 1,2,3]$ $\mathcal{P}[2,3,4,5]$. It is worth noticing that $z_{23}^{2}$ in numerator will cancel the $z_{23}^{2}$ in the denominator of $\mathcal{I}_{n}^{\mathrm{CHY}}$.

- With fixed poles $s_{n 1}$ and $s_{n 12}$, the integrand is given by $\mathcal{I}_{n}^{\mathrm{CHY}}(\{1,2, \ldots, n\})$ $\mathrm{P}[\mathrm{n}-1, \mathrm{n}, 1,2] \mathcal{P}[n-1, n, 2,3]$. For this case, pole $s_{n 1}$ is contained in the pole $s_{n 12}$.

The above examples have only two poles and it is easy to check that the numerator of the final expression is 1 . Thus we can check our claim easily using the mapping rule (2.2). However, when we fix three or more poles, something interesting happens: the numerator of the final expression could be nontrivial. For example, with the signature $s_{12} s_{123} s_{1234}$ at eight points, after applying the rule (2.11) we get

$$
\frac{z_{81}}{z_{12}^{2} z_{23} z_{34} z_{45} z_{56}^{2} z_{67}^{2} z_{78}^{2} z_{84} z_{15} z_{83} z_{14} z_{82} z_{13}} .
$$


Since the numerator is not 1 , we cannot apply the mapping rule directly. To overcome the problem, we need to use the following identity

$$
\frac{z_{a b} z_{d c}}{z_{a c} z_{b c}}=\frac{z_{a d}}{z_{a c}}-\frac{z_{b d}}{z_{b c}} .
$$

Applying this to our case with $z_{a b}=z_{81}$, we need to determine $c, d$. There are some conditions. Firstly the degree of factor $z_{d c}$ in the denominator of the original expression can only be zero or one. Thus, after multiplying $1=\frac{z_{d c}}{z_{d c}}$ and then using identity (2.13), we will not end up with factor $z_{d c}$ having degree larger than two in denominator. Secondly, we should require the original expression to have factors $z_{a c}, z_{b c}$ in its denominator to produce the left handed side of (2.13). Finally, we should require the original expression to have factors $z_{a d}$ and $z_{b d}$ in the denominator to cancel the corresponding factor in the numerator after using (2.13). If we can find such $d, c$, we can trivialize the problem and then apply the mapping rule. For the example given in (2.12), it is easy to see that $d, c$ can be chosen from $\{2,3,4\}$. In fact, there are six possible choices and we have checked all of them. With the choice $c=2, d=3$ we have

$$
\begin{aligned}
& \frac{z_{81}}{z_{12}^{2} z_{23} z_{34} z_{45} z_{56}^{2} z_{67}^{2} z_{78}^{2} z_{84} z_{15} z_{83} z_{14} z_{82} z_{13}}=\frac{-1}{z_{12} z_{23}^{2} z_{34} z_{45} z_{56}^{2} z_{67}^{2} z_{78}^{2} z_{84} z_{15} z_{83} z_{14} z_{13}} \times \frac{z_{81} z_{32}}{z_{82} z_{12}} \\
& =\frac{-1}{z_{12} z_{23}^{2} z_{34} z_{45} z_{56}^{2} z_{67}^{2} z_{78}^{2} z_{84} z_{15} z_{83} z_{14} z_{13}}\left(\frac{z_{83}}{z_{82}}-\frac{z_{13}}{z_{12}}\right) \\
& =\frac{-1}{z_{12} z_{23}^{2} z_{34} z_{45} z_{56}^{2} z_{67}^{2} z_{78}^{2} z_{84} z_{15} z_{82} z_{14} z_{13}}+\frac{+1}{z_{12}^{2} z_{23}^{2} z_{34} z_{45} z_{56}^{2} z_{67}^{2} z_{78}^{2} z_{84} z_{15} z_{83} z_{14}}
\end{aligned}
$$

Using the mapping rule, we can calculate each term and sum them up. It is easy to check that they indeed give all terms with the above signature.

We can continue to deal with more complicated examples, for example, the one with signature $s_{12} s_{123} s_{1234} s_{12345}$ at 8 points. One more pole indicates that we need to multiply it by another factor as

$$
\begin{aligned}
& \frac{z_{81}}{z_{12}^{2} z_{23} z_{34} z_{45} z_{56}^{2} z_{67}^{2} z_{78}^{2} z_{84} z_{15} z_{83} z_{14} z_{82} z_{13}} \times \frac{z_{81} z_{56}}{z_{85} z_{16}} \\
& =\left(\frac{-1}{z_{12} z_{23}^{2} z_{34} z_{45} z_{56}^{2} z_{67}^{2} z_{78}^{2} z_{84} z_{15} z_{82} z_{14} z_{13}}+\frac{+1}{z_{12}^{2} z_{23}^{2} z_{34} z_{45} z_{56}^{2} z_{67}^{2} z_{78}^{2} z_{84} z_{15} z_{83} z_{14}}\right) \times \frac{z_{81} z_{56}}{z_{85} z_{16}}
\end{aligned}
$$

where we have used the result (2.14). Now we use the similar idea to decompose these two terms. For the first term we take $c=2, d=4$ and get

$$
\frac{-1}{z_{23}^{2} z_{34} z_{45} z_{56} z_{67}^{2} z_{78}^{2} z_{82} z_{15} z_{14} z_{13} z_{85} z_{16} z_{42}}+\frac{+1}{z_{23}^{2} z_{34} z_{45} z_{56} z_{67}^{2} z_{78}^{2} z_{84} z_{15} z_{12} z_{13} z_{85} z_{16} z_{42}} .
$$

For the second term, we take $c=5, d=4$ and get

$$
\frac{1}{z_{12}^{2} z_{23}^{2} z_{34} z_{45}^{2} z_{56} z_{67}^{2} z_{78}^{2} z_{85} z_{83} z_{14} z_{16}}+\frac{-1}{z_{12}^{2} z_{23}^{2} z_{34} z_{45}^{2} z_{56} z_{67}^{2} z_{78}^{2} z_{84} z_{83} z_{15} z_{16}} .
$$

Applying the mapping rule to the above four terms and summing them up, we do get all terms with the signature of four fixed poles. 


\section{All-Loop scattering equations}

In this section, we will discuss the general $m$-th loop scattering equations. First we will review the construction given in [28], then we present another comprehension of these equations from the perspective of higher dimensions. To establish the relation between $m$-th loop $n$-point scattering equations and tree-level scattering equations of $(n+2 m)$ points, we use the following convention: $k_{i}, i=1, \ldots, n$ for $n$ external momenta, while $k_{n+2 j-1}=-k_{n+2 j}$ with $j=1, \ldots, m$ for the $j$-th loop momentum. While we still impose $k_{i}^{2}=0$ for $i=1, \ldots, n$, loop momenta $k_{n+2 j-1}$ are general massive.

To derive loop-level scattering equations, we start with the $m$-th loop one-form

$$
P=\sum_{r=1}^{m} k_{n+2 r-1} \frac{\left(z_{n+2 r-1}-z_{n+2 r}\right) d z}{\left(z-z_{n+2 r-1}\right)\left(z-z_{n+2 r}\right)}+\sum_{i=1}^{n} k_{i} \frac{d z}{z-z_{i}}
$$

where $z_{i}$, with $i=1, \ldots, n$ are marked points for external legs while $z_{n+2 r-1}, z_{n+2 r}$ with $r=1, \ldots, m$ are new marked points for the pinching Riemann sphere. It is easy to see that $P^{2}$ contains double poles, thus we define

$$
S(z)=P^{2}-\sum_{r=1}^{m} k_{n+2 r-1}^{2} \frac{\left(z_{n+2 r-1}-z_{n+2 r}\right)^{2} d^{2} z}{\left(z-z_{n+2 r-1}\right)^{2}\left(z-z_{n+2 r}\right)^{2}},
$$

which contains only single poles at all marked points $z_{i}$ with $i=1,2 \ldots, n+2 m$. Evaluating these residues, we get

$$
\mathcal{S}_{a}=\sum_{j \neq a, 1}^{n} \frac{2 k_{a} \cdot k_{j}}{z_{a}-z_{j}}+\sum_{t=1}^{m}\left(\frac{2 k_{a} \cdot k_{n+2 t-1}}{z_{a}-z_{n+2 t-1}}+\frac{2 k_{a} \cdot k_{n+2 t}}{z_{a}-z_{n+2 t}}\right), \quad 1 \leq a \leq n
$$

for $n$ external marked points and

$$
\begin{aligned}
\mathcal{S}_{n+2 t-1} & =\sum_{a=1}^{n} \frac{2 k_{n+2 t-1} \cdot k_{a}}{z_{n+2 t-1}-z_{a}}+\sum_{s=1, s \neq t}^{m}\left(\frac{2 k_{n+2 t-1} \cdot k_{n+2 s-1}}{z_{n+2 t-1}-z_{n+2 s-1}}+\frac{2 k_{n+2 t-1} \cdot k_{n+2 s}}{z_{n+2 t-1}-z_{n+2 s}}\right), \\
\mathcal{S}_{n+2 t} & =\sum_{a=1}^{n} \frac{2 k_{n+2 t} \cdot k_{a}}{z_{n+2 t}-z_{a}}+\sum_{s=1, s \neq t}^{m}\left(\frac{2 k_{n+2 t} \cdot k_{n+2 s-1}}{z_{n+2 t}-z_{n+2 s-1}}+\frac{2 k_{n+2 t} \cdot k_{n+2 s}}{z_{n+2 t}-z_{n+2 s}}\right), \quad 1 \leq t \leq m
\end{aligned}
$$

for new marked points corresponding to the $t$-th loop momentum. These $(n+2 m)$ equations given in (3.3) and (3.4) are the $m$-th loop scattering equations we are looking for.

Now we compare these equations with the corresponding tree-level scattering equations of $(n+2 m)$-points given by

$$
\mathcal{S}_{a} \equiv \sum_{b \neq a} \frac{2 k_{a} \cdot k_{b}}{z_{a}-z_{b}}=0, \quad a=1,2, \ldots, n+2 m .
$$

They are exactly the same for $a=1, \ldots, n$, except the remaining $2 m$ momenta satisfying $k_{n+2 j-1}=-k_{n+2 j}$ (i.e., in the forward limit). However, for $a=n+1, \ldots, n+2 m$, terms of the form $\frac{2 k_{n+2 t-1} \cdot k_{n+2 t}}{z_{n+2 t-1}-z_{n+2 t}}$ in tree-level scattering equations have been dropped in the $m$-th loop scattering equations. The dropping of these terms can, in fact, be traced back to the 
numerator $\left(z_{n+2 r-1}-z_{n+2 r}\right)$ of the first term in (3.1). This difference is crucial and we will explain it later.

Having obtained loop-level scattering equations, let us check their Mobius covariance. Under the Mobius transformation $z^{\prime}=\frac{a z+b}{c z+d}$, one finds

$$
z_{i j}^{\prime}=\frac{(a d-b c)}{\left(c z_{i}+d\right)\left(c z_{j}+d\right)} z_{i j}
$$

thus it is easy to check that for $\mathcal{S}_{1 \leq a \leq n}$ we have

$$
\begin{aligned}
\mathcal{S}_{a} \rightarrow & \frac{\left(c z_{a}+d\right)}{(a d-b c)}\left\{\sum_{j \neq a, 1}^{n} \frac{2 k_{a} \cdot k_{j}\left(c z_{j}+d\right)}{z_{a}-z_{j}}\right. \\
& \left.+\sum_{t=1}^{m}\left(\frac{2 k_{a} \cdot k_{n+2 t-1}\left(c z_{n+2 t-1}+d\right)}{z_{a}-z_{n+2 t-1}}+\frac{2 k_{a} \cdot k_{n+2 t}\left(c z_{n+2 t}+d\right)}{z_{a}-z_{n+2 t}}\right)\right\} \\
= & \frac{\left(c z_{a}+d\right)}{(a d-b c)}\left\{\sum_{j \neq a, 1}^{n}\left(\frac{2 k_{a} \cdot k_{j}\left(c z_{a}+d\right)}{z_{a}-z_{j}}-2 k_{a} \cdot k_{j}\right)\right. \\
& \left.+\sum_{t=1}^{m}\left(\frac{2 k_{a} \cdot k_{n+2 t-1}\left(c z_{a}+d\right)}{z_{a}-z_{n+2 t-1}}-2 k_{a} \cdot k_{n+2 t-1}+\frac{2 k_{a} \cdot k_{n+2 t}\left(c z_{a}+d\right)}{z_{a}-z_{n+2 t}}-2 k_{a} \cdot k_{n+2 t}\right)\right\} \\
= & \frac{\left(c z_{a}+d\right)^{2}}{(a d-b c)} \mathcal{S}_{a},
\end{aligned}
$$

and for $\mathcal{S}_{n<a \leq n+2 m}$ we have

$$
\begin{aligned}
\mathcal{S}_{n+2 t-1} \rightarrow & \frac{\left(c z_{n+2 t-1}+d\right)}{(a d-b c)}\left\{\sum_{a=1}^{n} \frac{2 k_{n+2 t-1} \cdot k_{a}\left(c z_{a}+d\right)}{z_{n+2 t-1}-z_{a}}\right. \\
& \left.+\sum_{s=1, s \neq t}^{m}\left(\frac{2 k_{n+2 t-1} \cdot k_{n+2 s-1}\left(c z_{n+2 s-1}+d\right)}{z_{n+2 t-1}-z_{n+2 s-1}}+\frac{2 k_{n+2 t-1} \cdot k_{n+2 s}\left(c z_{n+2 s}+d\right)}{z_{n+2 t-1}-z_{n+2 s}}\right)\right\} \\
= & \frac{\left(c z_{n+2 t-1}+d\right)}{(a d-b c)}\left\{\sum_{a=1}^{n}\left(\frac{2 k_{n+2 t-1} \cdot k_{a}\left(c z_{n+2 t-1}+d\right)}{z_{n+2 t-1}-z_{a}}-2 k_{n+2 t-1} \cdot k_{a}\right)\right. \\
& +\sum_{s=1, s \neq t}^{m}\left(\frac{2 k_{n+2 t-1} \cdot k_{n+2 s-1}\left(c z_{n+2 t-1}+d\right)}{z_{n+2 t-1}-z_{n+2 s-1}}-2 k_{n+2 t-1} \cdot k_{n+2 s-1}\right. \\
& \left.\left.+\frac{2 k_{n+2 t-1} \cdot k_{n+2 s}\left(c z_{n+2 t-1}+d\right)}{z_{n+2 t-1}-z_{n+2 s}}-2 k_{n+2 t-1} \cdot k_{n+2 s}\right)\right\} \\
= & \frac{\left(c z_{n+2 t-1}+d\right)^{2}}{(a d-b c)} \mathcal{S}_{n+2 t-1}
\end{aligned}
$$

and similar for $\mathcal{S}_{n+2 t}$.

This covariance indicates that there are three relations among the $(n+2 m)$ scattering equations:

$$
\sum_{i=1}^{n+2 m} \mathcal{S}_{i}=0, \quad \sum_{i=1}^{n+2 m} z_{i} \mathcal{S}_{i}=0, \quad \sum_{i=1}^{n+2 m} z_{i}^{2} \mathcal{S}_{i}=0
$$


We would to emphasize that in the above calculations, we have used only the following three conditions: (1) massless condition $k_{i}^{2}=0$ for $i=1, \ldots, n$; (2) momentum conservation $\sum_{i=1}^{n} k_{i}=0$; (3) forward limit $k_{n+2 j-1}=-k_{n+2 j}$ for $j=1, \ldots, m$. In other words, we do not need to impose $k_{n+2 j-1}^{2}=0$, which is due to the dropped terms of the form $\frac{2 k_{n+2 t-1} \cdot k_{n+2 t}}{z_{n+2 t-1}-z_{n+2 t}}$. In fact, it can be easily checked that without dropping these terms, the second and third relations in (3.9) cannot satisfy the above three conditions.

Now let us try to understand the meaning of dropping terms of the form $\frac{2 k_{n+2 t-1} \cdot k_{n+2 t}}{z_{n+2 t-1}-z_{n+2 t}}$. It is obvious that if $k_{n+2 t-1} \cdot k_{n+2 t}=-k_{n+2 t}^{2}=0$, it will disappear automatically. However, since they are loop momenta we should not impose these conditions. To make these two aspects consistent with each other, a nice idea is to use the dimension reduction. Let us assume that all external momenta are in the $D$-dimensional spacetime, then we can treat massive momenta in the $D$-dimensional spacetime as massless momenta in the $(D+d)$ dimensional spacetime. This can be arranged because scattering equations do not specify the dimensions. In fact, using the idea, several groups have noticed that scattering equations for massive particles ${ }^{1}$ at tree-level first proposed by Naculich in [46] can be understood from this perspective. More explicitly, let us rewrite the $(D+d)$-dimensional scattering equations as

$$
\sum_{j \neq i} \frac{\widetilde{k}_{i} \cdot \widetilde{k}_{j}}{z_{i}-z_{j}}=\sum_{j \neq i} \frac{k_{i} \cdot k_{j}+\Delta_{i j}}{z_{i}-z_{j}}
$$

where each $(D+d)$-dimensional momentum $\widetilde{k}=k+\eta$ has been split into component $k$ in $D$-dimension and component $\eta$ in $d$-dimension, so $\Delta_{i j}=-\eta_{i} \cdot \eta_{j}$. It is easy to see that $\Delta_{i j}=\Delta_{j i}$ and $\sum_{j \neq i} \Delta_{i j}=\eta_{i} \cdot \eta_{i}$ by momentum conservation in $d$-dimension. Thus massless condition $k_{i}^{2}-\eta_{i}^{2}=0$ in $(D+d)$-dimension gives the mass $\sum_{j \neq i} \Delta_{i j}=m_{i}^{2}$ in $D$-dimension.

The above discussions lead us to a new understanding of these $m$-th loop scattering equations in D-dimension: they are the tree-level scattering equations of $(n+2 m)$-points under the forward limit, where $2 m$ momenta are massless in $(D+d)$-dimension while $n$ external momenta are massless in D-dimension. An immediate implication is that all contractions of the type $2 k_{n+2 t-1} \cdot k_{n+2 s-1}$ in (3.4) should be understood as contractions in $(D+d)$-dimension.

The new understanding of loop momenta in higher dimensions has led to an important application: since from the perspective of higher dimensions they are massless, we have effectively cut $m$ 's internal lines, so $m$-th loop Feynman diagrams open up to become connected tree-level Feynman diagrams. This idea has been used in [32] to construct one-loop CHY integrands of $\phi^{3}$ theory (see also [41-43]). A more extensive application of reducing loop problems to tree-level ones has been demonstrated in the $\mathcal{Q}$-cut construction [44] (see also [45]). In this paper, we will use the same idea to write down CHY loop integrands from the corresponding tree ones.

\footnotetext{
${ }^{1}$ Other related works for massive particles can be found in [15, 47-49].
} 
Having understood their similarity and connection with tree-level cases, it is natural to write down the integration formula for loop amplitudes as proposed in [28]

$$
\mathcal{A}_{m-\text { loop }}^{D}=\int \prod_{i=1}^{m} \frac{d^{D} \ell_{i}}{\ell_{i}^{2}} \mathcal{I}_{m-\text { loop }}^{(D+d)}
$$

with

$$
\mathcal{I}_{m-\text { loop }}^{(D+d)}=\int \frac{\left(\prod_{i=1}^{n+2 m} d z_{i}\right)}{d \omega}\left(z_{i j} z_{j k} z_{k i} \prod_{a \neq i, j, k}^{n+2 m} \delta\left(\mathcal{S}_{a}\right)\right) \mathcal{I}^{\mathrm{CHY}}
$$

Here we will explain (3.11) and (3.12). Firstly although loop momenta in (3.11) are in $D$-dimension, when we use the CHY-formula to calculate $\mathcal{I}_{m \text {-loop }}^{(D+d)}$ as given in (3.12), we should treat loop momenta as massless in $(D+d)$-dimension as explained above. Thus we use the notation $(D+d)$ to emphasize this point. Secondly, (3.12) is the familiar tree-level CHY formula of $(n+2 m)$-points, where $d \omega=\frac{d z_{r} d z_{s} d z_{t}}{z_{r s} z_{s t} z_{t r}}$ comes from gauge fixing three $z$ 's by $\mathrm{SL}(2, C)$ symmetry. While other parts are universal for all theories, the CHY-integrand $\mathcal{I}^{\mathrm{CHY}}$ varies for different theories. Thus we will mainly focus on the construction of $\mathcal{I}^{\mathrm{CHY}}$.

The construction of CHY-integrands needs to satisfy some constraints. One of the most important constraints is the Mobius invariance. To compensate the variation of the measure in (3.12), under the $\operatorname{SL}(2, C)$ transformation, $\mathcal{I}^{\mathrm{CHY}}$ should have the following transformation property

$$
\mathcal{I}^{\mathrm{CHY}} \rightarrow\left(\prod_{i=1}^{n+2 m} \frac{(a d-b c)^{2}}{\left(c z_{i}+d\right)^{4}}\right)^{-1} \mathcal{I}^{\mathrm{CHY}}
$$

A nice way to fulfil above transformation property is to construct various combinations carrying different weights as demonstrated in [1-5]. Two familiar factors with weight 2 are (more factors can be found in $[4,5]$ )

$$
C_{\mathrm{U}(N)}(z)=\left(\sum_{\alpha \in S_{n} / Z_{n}} \frac{\operatorname{Tr}\left(T^{\alpha(1)} \ldots T^{\alpha(n)}\right)}{z_{\alpha(1) \alpha(2)} \ldots z_{\alpha(n-1) \alpha(n)} z_{\alpha(n) \alpha(1)}}\right), \quad E(\epsilon, k, z)=\left(\mathrm{Pf}^{\prime} \Psi(k, \epsilon, z) .\right.
$$

Besides the weight conditions, there are other physical aspects, such as the soft limit, the factorization property, etc.

Although using dimension reduction, we have mapped the loop problem to a tree one in (3.12), the CHY-integrand $\mathcal{I}^{\mathrm{CHY}}$ is not exactly the same one as the tree-level $\mathrm{CHY}$ integrand we are familiar with. There are two major reasons. The first one is that since tree-level Feynman diagrams are obtained by cutting internal lines, there are many choices of lines to be cut, thus one needs to sum over all allowed insertions of $2 m$ extra legs (and possibly also over polarization states of extra legs if particles running along the loop are not scalars). This issue has been discussed for one-loop cases in [32, 41-43]. The second reason is more crucial: after cutting loop diagrams to trees, we do not get all allowed tree-level diagrams of $(n+2 m)$-points. For example, for one-loop amplitudes of massless theories, 


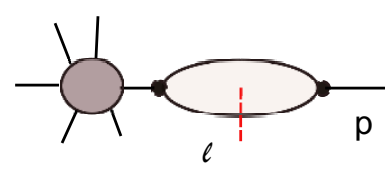

$(\mathrm{A}-1)$

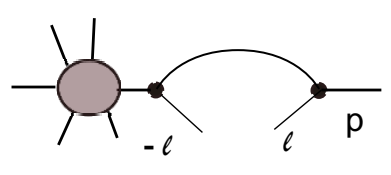

$(\mathrm{A}-2)$

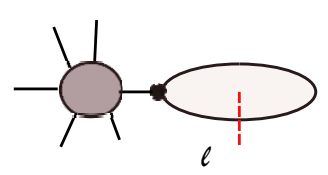

(B-1)

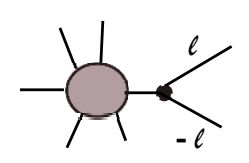

$(\mathrm{B}-2)$

Figure 3. The excluded one-loop Feynman diagrams of $\phi^{3}$ theory and their corresponding trees after the cut.

there are two types of diagrams we need to exclude: the tadpole diagrams (B-1) in figure 3 and the massless bubble diagrams (A-1) in figure 3. After reducing loop diagrams to trees, we should exclude these diagrams (A-2), (B-2) in figure 3 from allowed tree-level diagrams. These two types of tree diagrams are singular under the forward limit. Thus the exact CHY-integrand in (3.12) should be the one from trees after subtracting these divergent contributions.

However, to subtract these singular parts is very nontrivial. For some theories, for example, the supersymmetric theory, it has been shown in [50] that the singular forward limit disappears due to supersymmetry, ${ }^{2}$ so we do not need to take care of it. However, for pure Yang-Mills theory, theis subtraction in the CHY framework is not completely clear. Because of these subtleties, in this paper we will focus on planar loop integrands of colorordered bi-adjoint scalar $\phi^{3}$ theory. Although this case is simple, it is sufficient for one of the main purposes of the paper, i.e., to generalize the powerful mapping rule between CHY-integrands and Feynman diagrams given in [30-32] at the tree and one-loop levels.

\section{Two-loop CHY-integrand of $\phi^{3}$ theory}

Having discussed all-loop scattering equations, now we discuss how to write down all-loop CHY-integrands in (3.12), at least for the planar part of color-ordered bi-adjoint $\phi^{3}$ theory. For simplicity, we will use the two-loop example to demonstrate our strategy, but the idea should be easily generalized to all loops. The key strategy for loop CHY-integrands is to use the mapping rule found in papers [30-32]. Using the mapping rule, if we know the expressions from the field theory side through Feynman diagrams, we could find the corresponding CHY-integrands.

\subsection{Analysis of two-loop Feynman diagrams}

With this strategy, now we start to analyze color-ordered two-loop planar integrands obtained from Feynman diagrams. To have a clear picture of these integrands, let us classify planar two-loop Feynman diagrams of $\phi^{3}$ theory. It is easy to see that all diagrams can be separated into two types, i.e., type (A) and type (B) (see figure 4). The type (A) is the relatively trivial one as it is given by two sub one-loop diagrams. For these diagrams, we will use $T_{\left(n_{L} ; m_{u}, m_{d} ; n_{R}\right)}$ to denote them, where $n_{L}, n_{R}, m_{u}, m_{d}$ are numbers of external legs attached to the left sub one-loop, right sub one-loop, the upper part and the lower

\footnotetext{
${ }^{2}$ For other massless theories, recent $\mathcal{Q}$-cut construction in [44] has given a way to remove forward singularities by using the scale deformation.
} 

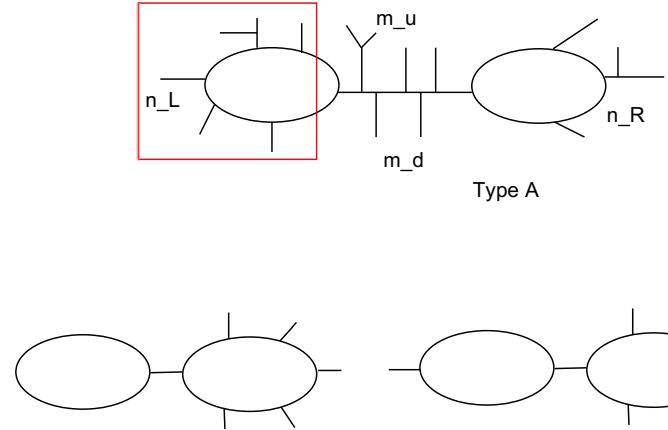

$(\mathrm{A}-1)$

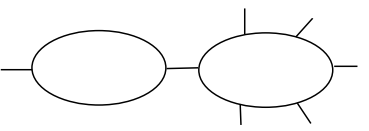

$(\mathrm{A}-2)$
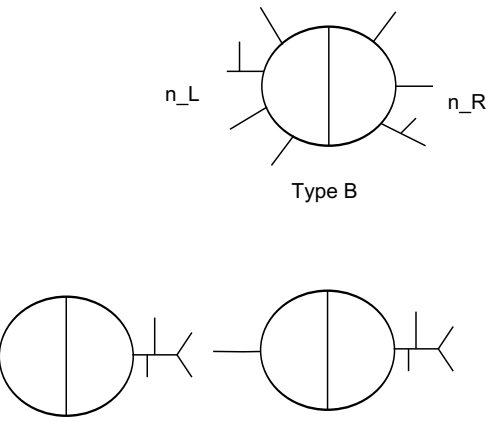

(B-2)

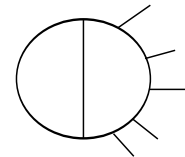

(B-3)

Figure 4. General planar two-loop Feynman diagrams Type (A) and Type (B) of $\phi^{3}$ theory. There are some special two-loop diagrams: (A-1) one-loop tadpole; (A-2) one-loop massless bubble; (B-1) two-loop tadpole; (B-2) two-loop massless bubble; and (B-3) Reducible two-loop diagrams.

part of the middle line. The type (B) is the nontrivial two-loop diagram with one mixed propagator. For these diagrams, we will use $T_{\left(n_{L} ; n_{R}\right)}$ to denote them, where $n_{L}, n_{R}$ are numbers of external legs attached to the left and the right parts.

Among these diagrams in figure 4, there are some singular two-loop diagrams, for which we will have more careful analysis. They are (see figure 4):

- When $n_{L}$ or $n_{R}$ of Type (A) is zero, we get the one-loop tadpole structure as given by $(\mathrm{A}-1)$.

- When $n_{L}$ or $n_{R}$ of Type (A) is 1 and all other external legs are grouped together and attached to another loop through only one vertex, we get the one-loop massless bubble structure as given by (A-2).

- When $n_{L}$ or $n_{R}$ of Type (B) is zero, we get the reducible two-loop structure as given by (B-3). For (B-3), when all external legs are grouped together and attached to the loop through only one vertex, we get the two-loop tadpole structure as given by (B-1).

- When $n_{L}$ or $n_{R}$ of Type (B) is 1 and all other external legs are grouped together and attached to another loop through only one vertex, we get the two-loop massless bubble structure as given by (B-2).

From general two-loop Feynman diagrams, we see that a two-loop integrands should be the sum of terms of the following two types ${ }^{3}$ (see diagrams (A) and (B) in figure 4)

$$
\begin{aligned}
\mathcal{I}_{A} & =\frac{1}{\mathcal{E}\left(\prod_{i}\left(\ell_{1}+K_{i}\right)^{2}\right)\left(\prod_{s}\left(\ell_{2}+K_{s}\right)^{2}\right)} \\
\mathcal{I}_{B} & =\frac{1}{\mathcal{E}\left(\prod_{i}\left(\ell_{1}+K_{i}\right)^{2}\right)\left(\prod_{s}\left(\ell_{2}+K_{s}\right)^{2}\right)\left(\ell_{1}-\ell_{2}+R\right)^{2}}
\end{aligned}
$$

\footnotetext{
${ }^{3}$ In our convention, the color ordering is clockwise. All external momenta are incoming while loop momenta will run along the clockwise direction, so when we cut internal propagator $\ell$ between leg 1 and leg 2 , the ordering should be $(1,-\ell, \ell, 2)$, i.e, moving along the clockwise direction is translated to moving from the left to the right. Furthermore, the nontrivial mixed propagator will be $\left(\ell_{1}-\ell_{2}+R\right)^{2}$.
} 
where $\mathcal{E}$ is the product of poles involving only external momenta. To continue, similar to the one-loop case $[22,32]$, we perform the partial fraction using the following identity ${ }^{4}$

$$
\frac{1}{\prod_{i=1}^{n} D_{i}}=\sum_{i=1}^{n} \frac{1}{D_{i} \prod_{j \neq i}\left(D_{j}-D_{i}\right)}
$$

and then shift the loop momenta. For the type $\mathcal{I}_{A}$, the partial fraction and loop momentum shift will give the standard form $\frac{1}{\ell_{1}^{2} \ell_{2}^{2}} \frac{1}{\prod P_{i}}$ with pole $P_{i}$ of the form $2 \ell_{i} \cdot\left(K_{j}-K_{i}\right)+\left(K_{j}-\right.$ $\left.K_{i}\right)^{2}=\left[\ell_{i}, k_{t_{1}}, \ldots, k_{t_{m}}\right]$ (see the notation (2.1)) when $K_{j}-K_{i}=\sum_{i=1}^{m} k_{t_{i}}$. These poles are the familiar ones appearing in the mapping rule $(2.2)^{5}$ at tree and one-loop levels. Thus it will not be so surprising that using the same mapping rule reviewed in section two we can easily read off the corresponding expressions given a CHY-integrand.

However, for the type $\mathcal{I}_{B}$, it is not so simple because now we have a mixed propagator ${ }^{6}$ $\left(\ell_{1}-\ell_{2}+R\right)^{2}$. When we do the partial fraction of $\ell_{1}$, should we include the mixed propagator $\left(\ell_{1}-\ell_{2}+R\right)^{2}$ in (4.2)? It is easy to see that if we include the mixed propagator, then we will have terms of the form $\frac{1}{\left(\ell_{1}-\ell_{2}+R\right)^{2}} \frac{1}{\prod_{i}\left(\left(\ell_{1}+K_{i}\right)^{2}-\left(\ell_{1}-\ell_{2}+R\right)^{2}\right)}$. To get the standard $\frac{1}{\ell_{1}^{2} \ell_{2}^{2}}$ factor in (3.11), we need to shift $\ell_{1}=\widehat{\ell}_{1}+\ell_{2}-R$. Although there is nothing wrong with this manipulation, the final pole $\left(\left(\ell_{1}+K_{i}\right)^{2}-\left(\ell_{1}-\ell_{2}+R\right)^{2}\right)$ will become $2 \widehat{\ell}_{1} \cdot\left(\ell_{2}-R+\right.$ $K)^{2}+\left(\ell_{2}-R+K\right)^{2}$, where although we have linearized $\widehat{\ell}_{1}, \ell_{2}^{2}$ will appear. The appearance of $\ell_{2}^{2}$ will make the next partial fraction of $\ell_{2}$ very complicated. Furthermore, the mapping rule succeeded at tree and one-loop levels cannot cooperate the term $\ell_{2}^{2}$. To avoid these difficulties, one possible way is to exclude the mixed propagator when we do the partial fraction for both loop momenta $\ell_{1}$ and $\ell_{2}$, then we will reach the sum of terms of the form $\frac{1}{\ell_{1}^{2} \ell_{2}^{2}\left(\ell_{1}-\ell_{2}+R\right)^{2} \prod_{i} \prod_{j=1,2}\left[\ell_{j}, k_{i_{1}}, \ldots, k_{i_{m}}\right]}$. Although the linearized poles fit the mapping rule, the remaining mixed propagator $\left(\ell_{1}-\ell_{2}+R\right)^{2}$ does not.

Is there a framework such that both features mentioned in the previous paragraph (i.e., the partial fraction without the mixed propagator and the applicability of the mapping rule) can be preserved? The answer is yes if we lift the massive loop momenta in $D$-dimension to massless loop momenta in $(D+d)$-dimension as discussed in previous section. As explained in the paper [44], the procedure of partial fraction can be understood as taking the residues of poles containing dimensionally deformed loop momenta. More explicitly, let us lift the loop momenta from $D$-dimension to $(D+d)$-dimension $\ell_{i} \rightarrow \widetilde{\ell}_{i}=\ell_{i}+\eta_{i}$. Under this deformation, we have

$$
\left(\ell_{i}+P\right)^{2} \rightarrow\left(\widetilde{\ell}_{i}+P\right)^{2}=\left(\ell_{i}+P\right)^{2}-\eta_{i}^{2} \equiv\left(\ell_{i}+P\right)^{2}+z_{i}
$$

as well as

$$
\left(\ell_{1}-\ell_{2}+R\right)^{2} \rightarrow\left(\tilde{\ell}_{1}-\tilde{\ell}_{2}+R\right)^{2}=\left(\ell_{1}-\ell_{2}+R\right)^{2}-\left(\eta_{1}-\eta_{2}\right)^{2}
$$

\footnotetext{
${ }^{4}$ The integrand of the type (B-3) in figure 4 is given by $\frac{1}{\ell_{1}^{2}\left(\prod_{i=1}^{m}\left(\ell_{1}+P_{i}\right)^{2}\right) \ell_{1}^{2}\left(\ell_{1}-\ell_{2}\right)^{2} \ell_{2}^{2}}$. The appearance of $\left(\ell_{1}^{2}\right)^{2}$ will make the partial fraction tricky. We will discuss these contributions later. Similar thing happens to the type (A-1).

${ }^{5}$ It is also worth noticing that it is these contractions $2 k_{i} \cdot k_{j}$ that appear in the numerators of scattering equations.

${ }^{6}$ For a two-loop planar diagram, there is at most one mixed propagator.
} 
As long as $d \geq 2$, we have the freedom to make different choices for $\left(\eta_{1}-\eta_{2}\right)^{2}$ while keeping $-\eta_{i}^{2}=z_{i}$ invariant. In [44], the choice is to treat $-\left(\eta_{1}-\eta_{2}\right)^{2}=z_{3}$ as a new independent variable, while for the current paper, we will choose $-\left(\eta_{1}-\eta_{2}\right)^{2}=0$. This can be achieved, for example, by taking

$$
\eta_{1}=(x+i y, x-i y), \quad \eta_{2}=(i x+z, i x-z)
$$

with

$$
\begin{aligned}
\left\{x=-\frac{i\left(z_{1}-z_{2}\right)}{\sqrt{(8+8 i) z_{1}-(8-8 i) z_{2}}}, \quad y\right. & =-\frac{i\left((2+i) z_{1}+i z_{2}\right)}{\sqrt{(8-8 i) z_{2}-(8+8 i) z_{1}}}, \\
z & \left.=\frac{z_{1}+(1+2 i) z_{2}}{2 \sqrt{2} \sqrt{(-1-i)\left(z_{1}+i z_{2}\right)}}\right\}
\end{aligned}
$$

thus $\left(\eta_{1}-\eta_{2}\right)^{2}=0$ for all $z_{1}, z_{2}$. Under this choice

$$
\begin{aligned}
\mathcal{I}_{B}\left(z_{1}, z_{2}\right) & =\frac{1}{\mathcal{E}\left(\ell_{1}-\ell_{2}+R\right)^{2}} T_{1}\left(z_{1}\right) T_{2}\left(z_{2}\right), \\
T_{1}\left(z_{1}\right) & =\frac{1}{\prod_{i}\left(\left(\ell_{1}+K_{i}\right)^{2}+z_{1}\right)}, \quad T_{2}\left(z_{2}\right)=\frac{1}{\prod_{s}\left(\left(\ell_{2}+K_{s}\right)^{2}+z_{2}\right)} .
\end{aligned}
$$

It is easy to see that using the contour integration $\oint \frac{d z_{1}}{z_{1}} T_{1}\left(z_{1}\right)$ we can write ${ }^{7}$

$$
\begin{aligned}
T_{1}\left(z_{1}=0\right) & =\sum_{i} \frac{1}{\left(\ell_{1}+K_{i}\right)^{2}} \frac{1}{\prod_{j \neq i}\left(\left(\ell_{1}+K_{j}\right)^{2}-\left(\ell_{1}+K_{i}\right)^{2}\right)} \\
& \sim \sum_{i} \frac{1}{\ell_{1}^{2}} \frac{1}{\prod_{j \neq i}\left(2 \ell_{1} \cdot\left(K_{j}-K_{i}\right)+\left(K_{j}-K_{i}\right)^{2}\right)}
\end{aligned}
$$

where in the second line we have shifted the loop momentum to make it have the standard form, which is legitimate under some proper regularization of loop integration (such as the dimensional regularization). Similar expression for $T_{2}\left(z_{2}=0\right)$ can be written down as well. The above manipulation is nothing but the partial fraction where the mixed propagator $\left(\ell_{1}-\ell_{2}+R\right)^{2}$ is not altered, which is exactly what we want. Furthermore, locations of poles impose on-shell conditions $\widetilde{\ell}_{i}^{2}=0 i=1,2$, thus the mixed propagator can be written as

$$
\begin{aligned}
\left(\ell_{1}-\ell_{2}+R\right)^{2}=\left(\tilde{\ell}_{1}-\tilde{\ell}_{2}+R\right)^{2}=-2 \tilde{\ell}_{1} \cdot \tilde{\ell}_{2}+R^{2}+2 R \cdot\left(\tilde{\ell}_{1}-\tilde{\ell}_{2}\right) & =\left[\tilde{\ell}_{1},-\tilde{\ell}_{2}, r_{1}, \ldots, r_{m}\right], \\
R & =\sum_{i} k_{r_{i}}
\end{aligned}
$$

which is exactly the correct pole structure given in the mapping rule (2.2).

Overall, under this new perspective, the two-loop planar integrand can be written as the sum of the following terms ${ }^{8}$

$$
\left.\frac{1}{\ell_{1}^{2} \ell_{2}^{2}}\left\{\sum_{i, s} \frac{1}{\widetilde{\mathcal{E}} \prod_{j \neq i}\left[\widetilde{\ell}_{1}, K_{j}-K_{i}\right] \prod_{t \neq s}\left[\widetilde{\ell}_{2}, K_{t}-K_{s}\right]^{2}}\right\}\right|_{\widetilde{\ell}_{1}^{2}=\widetilde{\ell}_{2}^{2}=0}
$$

\footnotetext{
${ }^{7}$ For this simple case, there is no residue at $z_{1}=\infty$.

${ }^{8}$ Again the form (4.10) must not contain contributions from the reducible two-loop diagrams (see type (B-3) in figure 4), for which we will discuss separately.
} 
where $\widetilde{\mathcal{E}}$ is $\mathcal{E}$ for $\mathcal{I}_{A}$ and $\mathcal{E}\left[\widetilde{\ell}_{1},-\widetilde{\ell}_{2}, R\right]$ for $\mathcal{I}_{B}$. From (4.10) it is clear that the calculation of two-loop integrands is reduced to the calculation of the part inside the curly braces. What is the physical picture for these terms? The on-shell conditions $\widetilde{\ell}_{1}^{2}=\widetilde{\ell}_{2}^{2}=0$ mean that we have cut two loop momenta to make them on-shell, thus two-loop diagrams open up to become tree diagrams with 4 extra legs having momenta $-\widetilde{\ell}_{1}, \widetilde{\ell}_{1},-\widetilde{\ell}_{2}, \widetilde{\ell}_{2}$. However, as we have discussed before, not all tree diagrams with $(n+4)$-legs can be obtained by this way, especially these coming from one- and two-loop tadpoles and massless bubbles (see figure 4). We will discuss this problem in the next subsection.

\subsection{Special Feynman diagrams}

In this subsection, we focus on the special diagrams given in figure 4. Among them, tadpoles and massless bubbles are singular, thus we should remove the corresponding contributions of these tree diagrams, which are obtained from these singular two-loop diagrams after cutting two internal propagators, from (4.10). In order to do so, we need to have a better understanding of these tree diagrams.

Let us start from the one-loop tadpoles (A-1) and massless bubbles (A-2) in figure 4 . Depending on whether the left or right sub one-loop are tadpoles or massless bubbles, we have four different combinations, which are given by four boxed corners in figure 5. For the upper-left corner, it is the left sub one-loop having tadpole structure while the right sub one-loop can have an arbitrary structure. After cutting two loop propagators, we get the corresponding tree diagrams with $(n+4)$-legs. However, all these diagrams have a common feature: all of them contain the pole $s_{\left(-\ell_{1}\right) \ell_{1}}$. We will call it the signature of the tadpole structure. For the lower-right corner, the left sub one-loop has the massless bubble structure while the right sub one-loop can have an arbitrary structure. After cutting two loop propagators, we get the corresponding tree diagrams with the following signature of the massless bubble structure: either containing pole $s_{\left(-\ell_{1}\right) p} s_{\left(-\ell_{1}\right) \ell_{1} p}$ or containing pole $s_{\ell_{1} p} s_{\left(-\ell_{1}\right) \ell_{1} p}$ with $p$, the massless leg. Similar analysis can be done for the upper-right corner where the right sub one-loop has the massless bubble structure and the lower-left corner where the right sub one-loop has the tadpole structure.

The above four corners have encoded all singular behaviors of the sub one-loop structure in two-loop diagrams. However, they are not completely decouple from each other. For example, we can have the special case where both left and right sub one-loops have the tadpole structure. This has been given in the middle between the upper-left corner and the lower-left corner in figure 5. The signature of the corresponding tree diagrams is the appearance of poles $s_{\left(-\ell_{1}\right) \ell_{1}}$ and $s_{\left(-\ell_{2}\right) \ell_{2}}$ at same time. Similar story happens for each pair of corners next to each other and we have listed all of them in figure 5.

Having understood the one-loop tadpole and massless bubble singularities, now we consider the two-loop tadpole and massless bubble singularities. For the two-loop massless bubble given in figure 6 , depending on different combinations of cuts, we have four different tree diagrams. Among these four cases, the forward pairs $\left(-\ell_{1}, \ell_{1}\right)$ and $\left(-\ell_{2}, \ell_{2}\right)$ are next to each other only in two of them. The signatures of these four cases are $s_{\left(-\ell_{1}\right) \ell_{2}} s_{P \ell_{1}} s_{Q\left(-\ell_{2}\right)}$, $s_{Q\left(-\ell_{1}\right) \ell_{2}} s_{P \ell_{1}} s_{Q \ell_{2}}, s_{p\left(-\ell_{1}\right) \ell_{2}} s_{P\left(-\ell_{1}\right)} s_{Q\left(-\ell_{2}\right)}$ and $s_{\ell_{1}\left(-\ell_{2}\right)} s_{P\left(-\ell_{1}\right)} s_{Q \ell_{2}}$ with $P+Q=0$. Furthermore, depending on whether $P$ or $Q$ is massless, we need to add another pole $s_{P}$ or $s_{Q}$. 

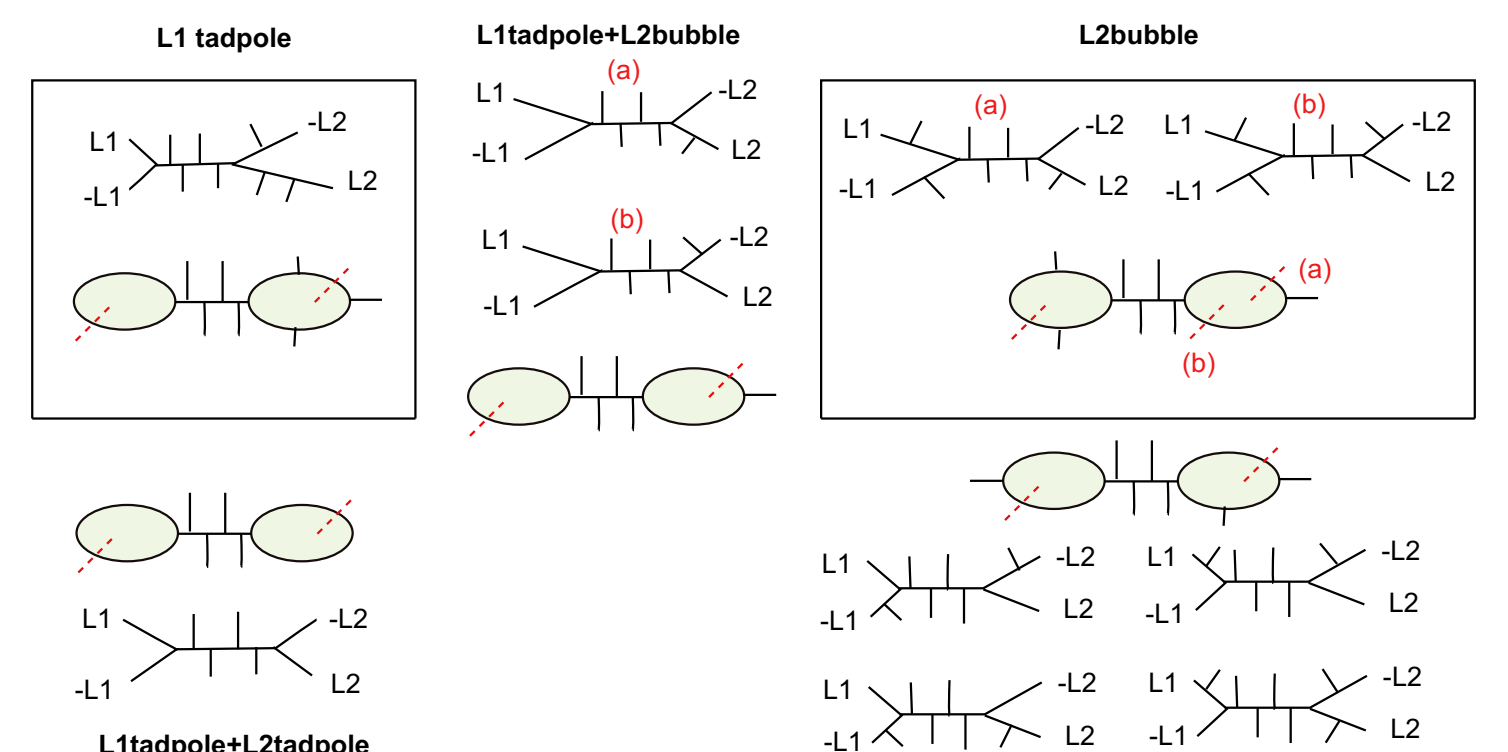

L1tadpole+L2tadpole

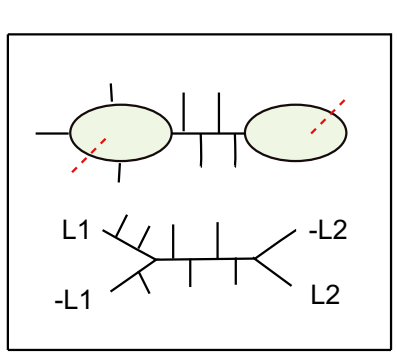

L2 tadpole

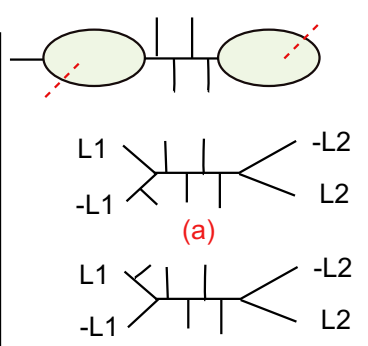

(b)

L1bubble+L2tadpole
L1bubble+L2bubble

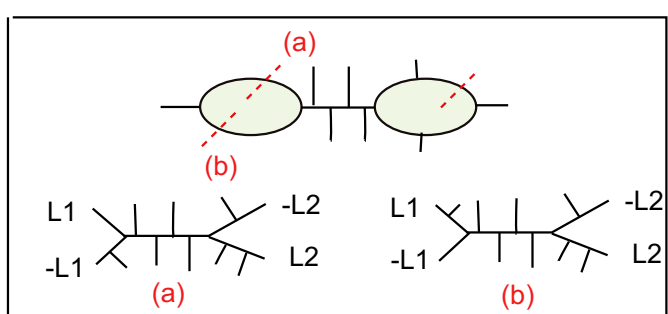

L1bubble

Figure 5. Singular contributions from the one-loop tadpoles and massless bubbles. At the four corners, we have four generic cases. For example, the corner L1-tadpole means that the left oneloop is a tadpole while the right one-loop can be generic. Each pair of adjacent corners has an intersection. For example, between the corner L1-tadpole and the corner L2-bubble we will have the diagram where the left one-loop is a tadpole and the right one-loop is a massless bubble. For each loop diagram, we have also drawn the corresponding tree diagrams after the cut. These pictures will be very useful when we discuss how to write down the CHY-integrand.

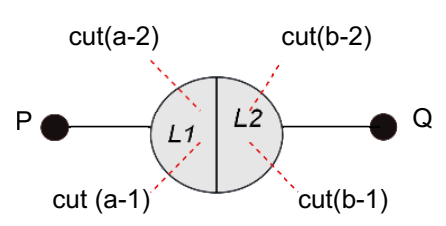

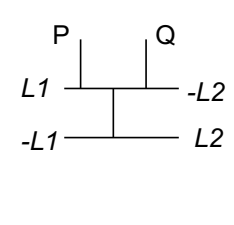

$(\mathrm{a} 1+\mathrm{b} 1)$

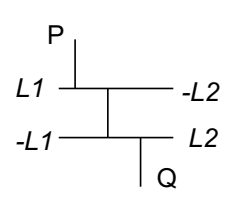

$(\mathrm{a} 1+\mathrm{b} 2)$

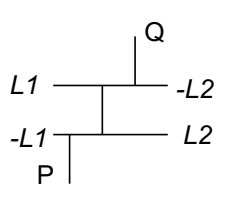

$(\mathrm{a} 2+\mathrm{b} 1)$

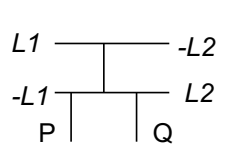

$(a 2+b 2)$

Figure 6. The two-loop massless bubble and its corresponding tree diagrams after various combinations of cuts.

To discuss the two-loop tadpole, let us start with (B-3) in figure 4. Since all external legs are attached to one side, the integrand will have the form (see (A-1) of figure 7)

$$
\frac{1}{\ell_{1}^{2}\left(\prod_{i=1}^{m}\left(\ell_{1}+P_{i}\right)\right) \ell_{1}^{2}\left(\ell_{1}-\ell_{2}\right)^{2} \ell_{2}^{2}}
$$




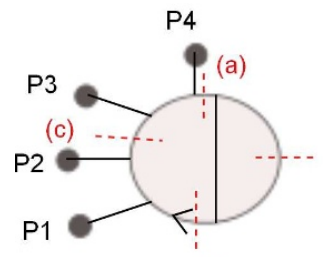

(b)

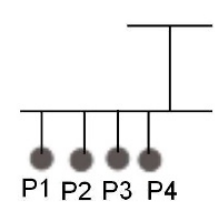

(a)

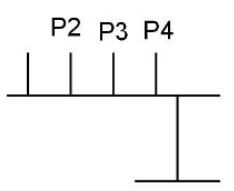

(b)

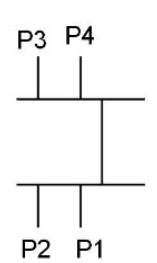

(c)

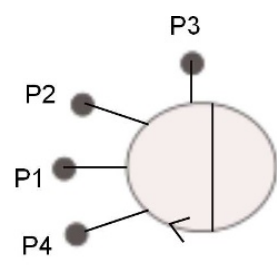

$(\mathrm{A}-2)$

Figure 7. The reducible two-loop diagram (A-1) and its corresponding tree diagrams (a), (b), (c) after cuts. The two-loop diagram (A-2) are obtained from (A-1) by a cyclic permutation. (A-1) and (A-2) give different contributions and we should sum over all cyclic permutations, plus the symmetrization between $\ell_{1}, \ell_{2}$.

where the appearance of $\left(\ell_{1}^{2}\right)^{2}$ makes the naive partial fraction to (4.1) problematic. Thus we should not expect to reduce these contributions to the form (4.10). Then how to deal with them? One hint is to rearrange (4.11) as

$$
\frac{1}{\ell_{1}^{2}\left(\ell_{1}-\ell_{2}\right)^{2} \ell_{2}^{2}}\left\{\frac{1}{\ell_{1}^{2}\left(\prod_{i=1}^{m}\left(\ell_{1}+P_{i}\right)\right)}\right\}
$$

then the part inside the curly braces is nothing but the familiar one-loop integrand. However, there is one subtlety regarding the choice of loop momentum $\ell_{1}$. With the convention of (A-1) and (A-2) of figure 7, it is easy to see that although when rewriting to the form (4.12), both produce one-loop integrands with the same color ordering, these two one-loop integrands are not the same since they have different conventions of loop momentum $\ell_{1}$ inherited from two-loop diagrams (although they are related by loop momentum shifting). With the above understanding, we can write two-loop integrands obtained from the type (B-3) in figure 4 as

$\mathcal{I}_{B_{3}}^{2-\text { loop }}=\left\{\frac{1}{\ell_{1}^{2}\left(\ell_{1}-\ell_{2}\right)^{2} \ell_{2}^{2}}\left\{\mathcal{I}^{1-\text { loop }}\left(1,2, \ldots, n, \ell_{1}\right)+\operatorname{cyclic} \operatorname{permu}\{1,2, \ldots, n\}\right\}\right\}+\left\{\ell_{1} \leftrightarrow \ell_{2}\right\}$

Now we give some explanations for (4.13). Firstly, in each one-loop diagram of $\mathcal{I}^{1-\text { loop }}\left(1,2, \ldots, n, \ell_{1}\right)$, the loop propagator at the right of the vertex where leg 1 is connected to, is defined to be $\ell_{1}$. Secondly, the two-loop tadpole diagram (B-1) in figure 4 is reduced to the one-loop tadpole diagram, thus if we exclude these contributions from tadpole diagrams in $\mathcal{I}^{1-\text { loop }}\left(1,2, \ldots, n, \ell_{1}\right)$, we have excluded the two-loop tadpole contributions. Thirdly, since we have reduced the calculation of $\mathcal{I}_{B_{3}}^{2-\text { loop }}$ to the one-loop case, we can regard them as the known data. Thus when we try to find the CHY-construction of two-loop integrands in (4.10), we can exclude $\mathcal{I}_{B_{3}}^{2-\text { loop }}$ part. The complete planar two-loop integrand will be the sum of (4.10) and (4.13). This will be the strategy we adopt in the later part of the paper, although in subsection 4.4 we do give a CHY-construction of the $\mathcal{I}_{B_{3}}^{2-\text { loop }}$ part as the soft limit of the corresponding theory with $(n+5)$-points. 


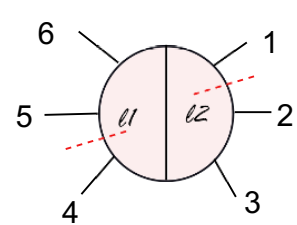

(a-1)

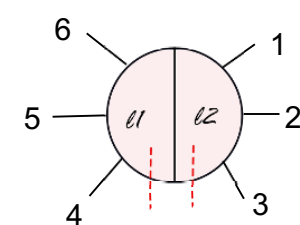

(b-1)

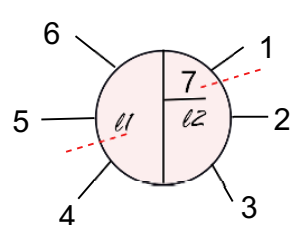

(c-1)

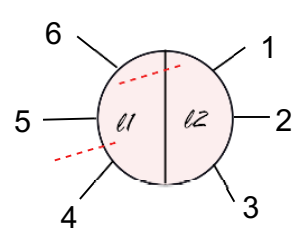

(d-1)

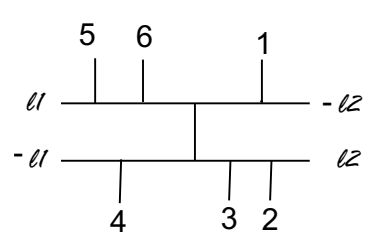

(a-2)

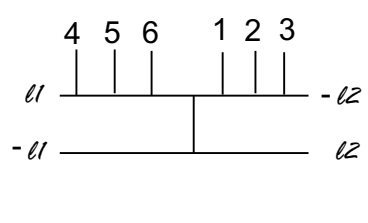

$(b-2)$

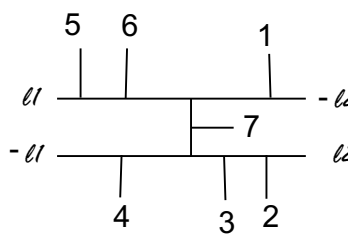

(c-2)

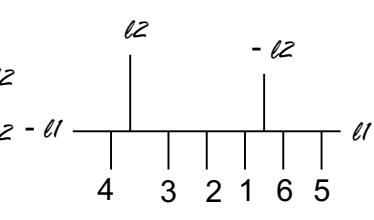

(d-2)

Figure 8. Planar two-loop diagrams with two cuts and their corresponding color ordered tree diagrams. In (a),(b), $-\ell_{i}, \ell_{i}$ are next to each other while in $(\mathrm{c}),(\mathrm{d})$ they are not.

\subsection{The construction of CHY-integrand}

Having reduced the problem of figuring out loop integrands to that of tree diagrams in (4.10) (after excluding the $\mathcal{I}_{B_{3}}^{2-l o o p}$ part), we need to determine which tree diagrams to consider. Since these tree diagrams are obtained from planar two-loop diagrams by cutting two internal propagators, we can get the general picture by speculating the figure 8 . By checking different combinations of two cuts, such as those in (a-1) and (b-1), we can see that in the resulted color ordered tree diagrams (such as these in (a-2) and (b-2)), $-\ell_{1}$ is always next to $\ell_{1}$ (similar for the pair $-\ell_{2}, \ell_{2}$ ). This pattern no longer holds for non-planar twoloop diagrams (see (c-2)) or when the mixed propagator is cut (see (d-2)). Observing this we conclude that the obtained tree diagrams are those obtained from the original color-ordered $n$-point tree diagrams after inserting two pairs $\left(-\ell_{i}, \ell_{i}\right)$ consistently into all possible locations. More explicitly, we will have the following two types of ordered diagrams of $(n+4)$-points:

- Type (I): there are $2 n$ terms of the following ordering

$$
\mathcal{O}_{j} \equiv\left\{1, \ldots, j,\left(-\widetilde{\ell}_{1}, \widetilde{\ell}_{1}\right),\left(-\widetilde{\ell}_{2}, \widetilde{\ell}_{2}\right), j+1, \ldots, n\right\}
$$

with $j=1,2, \ldots, n$ (plus also the symmetrization of $\left.\tilde{\ell}_{1} \leftrightarrow \tilde{\ell}_{2}\right) \cdot{ }^{9}$

- Type (II): there are $n(n-1)$ of them of the following ordering

$$
\mathcal{O}_{j k} \equiv\left\{1, \ldots, j,\left(-\ell_{1}, \ell_{1}\right), j+1, \ldots, k,\left(-\ell_{2}, \ell_{2}\right), k+1, \ldots, n\right\}
$$

with $1 \leq j<k \leq n$ (plus also the symmetrization of $\widetilde{\ell}_{1} \leftrightarrow \widetilde{\ell}_{2}$ ).

Having found the related color-ordered tree amplitudes, we know immediately that the part inside the curly braces of (4.10) is the sum of these color-ordered tree amplitudes of types

\footnotetext{
${ }^{9}$ The symmetrization is necessary since there is no canonical definition of loop variables at two loop.
} 
(I) and (II), after removing possible forward singularities and the $\mathcal{I}_{B_{3}}^{2-\text { loop }}$ part contained in them. Thus the desired CHY-integrand $\mathcal{I}^{\mathrm{CHY}}$ in (3.12) should produce these contributions. To find it, we need to use the mapping rule established in [30-32]. Now we discuss them one by one.

\subsubsection{The CHY-integrand for ordering $\mathcal{O}_{j k}$}

Having the above general discussions, now we determine the CHY-integrand for each ordering in (4.14) and (4.15). Let us start with the ordering $\mathcal{O}_{j k}$. With this ordering, the full tree-level amplitude is given by the following CHY-integrand

$$
T_{j k}=\frac{1}{z_{12}^{2} \ldots z_{j\left(-\ell_{1}\right)}^{2} z_{\left(-\ell_{1}\right) \ell_{1}}^{2} z_{\ell_{1}(j+1)}^{2} \ldots z_{k\left(-\ell_{2}\right)}^{2} z_{\left(-\ell_{2}\right) \ell_{2}}^{2} z_{\ell_{2}(k+1)}^{2} \cdots z_{n 1}^{2}} .
$$

Now we consider various forward limits, which can be produced in this ordering. For this purpose, figure 5, figure 6 and figure 7 are very useful. From them, we see that this ordering may contain the following singularities:

- Firstly it may contain the $\ell_{1}$-tadpole singularity, i.e., the pole $s_{\left(-\ell_{1}\right) \ell_{1}}$. These tree diagrams are obtained from the CHY-integrand

$$
T_{j k ; t_{1}}=\frac{1}{z_{12}^{2} \ldots z_{j\left(-\ell_{1}\right)} z_{j \ell_{1}} z_{\left(-\ell_{1}\right) \ell_{1}}^{2}{\underline{\left(-\ell_{1}\right)(j+1)}}_{\ell_{1}(j+1)}^{z_{(-1)}} z_{k\left(-\ell_{2}\right)}^{2} z_{\left(-\ell_{2}\right) \ell_{2}}^{2} z_{\ell_{2}(k+1)}^{2} \ldots z_{n 1}^{2}}
$$

where we use $t_{1}$ to denote the $\ell_{1}$-tadpole singularity and underlines to emphasize the altered factors. We can write (4.17) in a more compact way by using the rule (2.11)

$$
T_{j k ; t_{1}}=T_{i j} \mathcal{P}\left[j,-\ell_{1}, \ell_{1}, j+1\right]
$$

- Secondly it contains massless $\ell_{1}$-bubble singularities, i.e., those with pole $s_{j\left(-\ell_{1}\right)}$ $s_{j\left(-\ell_{1}\right) \ell_{1}}$ or $s_{\ell_{1}(j+1)} s_{\left(-\ell_{1}\right) \ell_{1}(j+1)}$. Using the rule $(2.11)$ we can write the corresponding CHY-integrands as

$$
T_{j k ; b_{1} j}=T_{j k} \mathcal{P}\left[j-1, j,-\ell_{1}, \ell_{1}\right] \mathcal{P}\left[j-1, j, \ell_{1}, j+1\right]
$$

and

$$
T_{j k ; b_{1}(j+1)}=T_{j k} \mathcal{P}\left[-\ell_{1}, \ell_{1}, j+1, j+2\right] \mathcal{P}\left[j,-\ell_{1}, j+1, j+2\right]
$$

where we use $b_{1}$ for massless bubble involving $\ell_{1}$ and $j$ to denote the massless bubble of the $j$-th leg.

We would like to emphasize one issue: the above three singularities are not compatible, i.e., they cannot appear at same time in a given tree diagram. Thus when we subtract their contributions, we should subtract all of them. 
- Similar considerations can be done for the $\ell_{2}$ part and we get the following three CHY-integrands

$$
\begin{aligned}
T_{j k ; t_{2}} & =T_{i j} \mathcal{P}\left[k,-\ell_{2}, \ell_{2}, k+1\right] \\
T_{j k ; b_{2} k} & =T_{j k} \mathcal{P}\left[k-1, k,-\ell_{2}, \ell_{2}\right] \mathcal{P}\left[k-1, k, \ell_{2}, k+1\right] \\
T_{j k ; b_{2}(k+1)} & =T_{j k} \mathcal{P}\left[-\ell_{2}, \ell_{2}, k+1, k+2\right] \mathcal{P}\left[k,-\ell_{2}, k+1, k+2\right]
\end{aligned}
$$

corresponding to one-loop tadpoles and massless bubbles of $\ell_{2}$.

- Now there is an important observation: the one-loop tadpole and massless bubble singularities of $\ell_{1}$ are (almost) compatible with those of $\ell_{2}$. Thus we will have the following nine $\mathrm{CHY}$-integrands, which produce tree diagrams containing both types of singularities. They are

$$
\begin{aligned}
T_{j k ; t_{1}, t_{2}} & =T_{i j} \mathcal{P}\left[j,-\ell_{1}, \ell_{1}, j+1\right] \mathcal{P}\left[k,-\ell_{2}, \ell_{2}, k+1\right], \\
T_{j k ; t_{1}, b_{2} k} & =T_{j k} \mathcal{P}\left[j,-\ell_{1}, \ell_{1}, j+1\right] \mathcal{P}\left[k-1, k,-\ell_{2}, \ell_{2}\right] \mathcal{P}\left[k-1, k, \ell_{2}, k+1\right], \\
T_{j k ; t_{1}, b_{2}(k+1)} & =T_{j k} \mathcal{P}\left[j,-\ell_{1}, \ell_{1}, j+1\right] \mathcal{P}\left[-\ell_{2}, \ell_{2}, k+1, k+2\right] \mathcal{P}\left[k,-\ell_{2}, k+1, k+2\right],
\end{aligned}
$$

and

$$
\begin{aligned}
T_{j k ; b_{1} j, t_{2}}= & T_{i j} \mathcal{P}\left[j-1, j,-\ell_{1}, \ell_{1}\right] \mathcal{P}\left[j-1, j, \ell_{1}, j+1\right] \mathcal{P}\left[k,-\ell_{2}, \ell_{2}, k+1\right], \\
T_{j k ; b_{1} j, b_{2} k}= & T_{j k} \mathcal{P}\left[j-1, j,-\ell_{1}, \ell_{1}\right] \mathcal{P}\left[j-1, j, \ell_{1}, j+1\right] \mathcal{P}\left[k-1, k,-\ell_{2}, \ell_{2}\right] \\
& \mathcal{P}\left[k-1, k, \ell_{2}, k+1\right], \\
T_{j k ; b_{1} j, b_{2}(k+1)}= & T_{j k} \mathcal{P}\left[j-1, j,-\ell_{1}, \ell_{1}\right] \mathcal{P}\left[j-1, j, \ell_{1}, j+1\right] \mathcal{P}\left[-\ell_{2}, \ell_{2}, k+1, k+2\right] \\
& \mathcal{P}\left[k,-\ell_{2}, k+1, k+2\right],
\end{aligned}
$$

and

$$
\begin{aligned}
T_{j k ; b_{1}(j+1), t_{2}}= & T_{i j} \mathcal{P}\left[-\ell_{1}, \ell_{1}, j+1, j+2\right] \mathcal{P}\left[j,-\ell_{1}, j+1, j+2\right] \mathcal{P}\left[k,-\ell_{2}, \ell_{2}, k+1\right], \\
T_{j k ; b_{1}(j+1), b_{2} k}= & T_{j k} \mathcal{P}\left[-\ell_{1}, \ell_{1}, j+1, j+2\right] \mathcal{P}\left[j,-\ell_{1}, j+1, j+2\right] \mathcal{P}\left[k-1, k,-\ell_{2}, \ell_{2}\right] \\
& \mathcal{P}\left[k-1, k, \ell_{2}, k+1\right], \\
T_{j k ; b_{1}(j+1), b_{2}(k+1)=} & T_{j k} \mathcal{P}\left[-\ell_{1}, \ell_{1}, j+1, j+2\right] \mathcal{P}\left[j,-\ell_{1}, j+1, j+2\right] \\
& \mathcal{P}\left[-\ell_{2}, \ell_{2}, k+1, k+2\right] \mathcal{P}\left[k,-\ell_{2}, k+1, k+2\right] .
\end{aligned}
$$

There is a warning: when there is only one leg between two pairs of loop momenta, among the above nine combinations, some cannot exist. More explicitly, when $k=$ $j+1$, the combination $T_{j k ; b_{1}(j+1), b_{2} k}$ cannot exist, while when $k=n, j=1$ the combination $T_{j k ; b_{1} j, b_{2}(k+1)}$ cannot exist.

The reason to discuss the compatibility is to avoid over-subtracting the singular part. For example, after we subtract $T_{j k ; t_{1}}$ and $T_{j k ; t_{2}}$ from $T_{j k}$, the part $T_{j k ; t_{1}, t_{2}}$ has been subtracted twice, thus we need to add the $T_{j k ; t_{1}, t_{2}}$ part for compensation. 
- Having excluded the one-loop singularities, we continue to remove two-loop massless bubble singularities. Although a bit away from our scope, let us start with the bubble structure, which includes the massive bubble. From figure 6, we see that the two-loop bubble structure will have five fixed poles. With the signature $s_{(j+1) \ldots k} s_{\ell_{1}(j+1) \ldots k} s_{(k+1) \ldots j} s_{\ell_{2}(k+1) \ldots j} s_{\ell_{2}(k+1) \ldots j\left(-\ell_{1}\right)}$, the corresponding CHYintegrand is

$$
\begin{aligned}
T_{j k ; 2 m 1}= & T_{j k} \mathcal{P}\left[\ell_{1}, j+1, k,-\ell_{2}\right] \mathcal{P}\left[-\ell_{1}, \ell_{1}, k,-\ell_{2}\right] \mathcal{P}\left[\ell_{2}, k+1, j,-\ell_{1}\right] \\
& \mathcal{P}\left[-\ell_{2}, \ell_{2}, j,-\ell_{1}\right] \mathcal{P}\left[-\ell_{2}, \ell_{2},-\ell_{1}, \ell_{1}\right]
\end{aligned}
$$

while with the signature $s_{(j+1) \ldots k} s_{(j+1) \ldots k\left(-\ell_{2}\right)} s_{(k+1) \ldots j} s_{(k+1) \ldots j\left(-\ell_{1}\right)} s_{\ell_{2}(k+1) \ldots j\left(-\ell_{1}\right)}$, the corresponding CHY-integrand is

$$
\begin{aligned}
T_{j k ; 2 m 2}= & T_{j k} \mathcal{P}\left[\ell_{1}, j+1, k,-\ell_{2}\right] \mathcal{P}\left[\ell_{1}, j+1,-\ell_{2}, \ell_{2}\right] \mathcal{P}\left[\ell_{2}, k+1, j,-\ell_{1}\right] \\
& \mathcal{P}\left[\ell_{2}, k+1,-\ell_{1}, \ell_{1}\right] \mathcal{P}\left[-\ell_{2}, \ell_{2},-\ell_{1}, \ell_{1}\right]
\end{aligned}
$$

These pole structures with five $s$-factors are generic. To get the massless bubble, pole $s_{(j+1) \ldots k}=s_{(k+1) \ldots j}$ must be zero. This can happen only when $k=j+1$ or $k=n, j=1$. For $k=j+1$, the signature of the $T_{j k ; 2 m 1}$ type reduces to $s_{\ell_{1}(j+1) \ldots k} s_{(k+1) \ldots j} s_{\ell_{2}(k+1) \ldots j} s_{\ell_{2}(k+1) \ldots j\left(-\ell_{1}\right)}$ and the corresponding CHY-integrand is

$$
T_{j k ; 2 m 1}=T_{j k} \mathcal{P}\left[-\ell_{1}, \ell_{1}, k,-\ell_{2}\right] \mathcal{P}\left[\ell_{2}, k+1, j,-\ell_{1}\right] \mathcal{P}\left[-\ell_{2}, \ell_{2}, j,-\ell_{1}\right] \mathcal{P}\left[-\ell_{2}, \ell_{2},-\ell_{1}, \ell_{1}\right]
$$

while the signature of the $T_{j k ; 2 m 2}$ type reduces $s_{(j+1) \ldots k\left(-\ell_{2}\right)} s_{(k+1) \ldots j} s_{(k+1) \ldots j\left(-\ell_{1}\right)}$ $s_{\ell_{2}(k+1) \ldots j\left(-\ell_{1}\right)}$ and the corresponding CHY-integrand is

$$
T_{j k ; 2 m 2}=T_{j k} \mathcal{P}\left[\ell_{1}, j+1,-\ell_{2}, \ell_{2}\right] \mathcal{P}\left[\ell_{2}, k+1, j,-\ell_{1}\right] \mathcal{P}\left[\ell_{2}, k+1,-\ell_{1}, \ell_{1}\right] \mathcal{P}\left[-\ell_{2}, \ell_{2},-\ell_{1}, \ell_{1}\right]
$$

These two expressions (4.35) and (4.36) can be obtained from (4.33) and (4.34) by trivially setting $k=j+1$ since $\left.\mathcal{P}\left[\ell_{1}, j+1, k,-\ell_{2}\right]\right|_{k=j+1}=1$. Similarly for the case $k=n, j=1$, the corresponding CHY-integrands can also be obtained from (4.33) and (4.34) by trivially setting $k=n, j=1$ since $\left.\mathcal{P}\left[\ell_{2}, k+1, j,-\ell_{1}\right]\right|_{k=n, j=1}=1$.

These two are not compatible to each other. They are also not compatible with one-loop tadpole and massless bubble singularities.

- The last piece we need to exclude is the (B-3) part in figure 4. From figure 7, we see that with the signature $s_{\ell_{1}(j+1) \ldots k} s_{(k+1) \ldots j\left(-\ell_{1}\right)} s_{\ell_{2}(k+1) \ldots j\left(-\ell_{1}\right)}$, the corresponding CHY-integrand is

$$
T_{j k ; B 31}=T_{j k} \mathcal{P}\left[-\ell_{1}, \ell_{1}, k,-\ell_{2}\right] \mathcal{P}\left[\ell_{2}, k+1,-\ell_{1}, \ell_{1}\right] \mathcal{P}\left[-\ell_{2}, \ell_{2},-\ell_{1}, \ell_{1}\right]
$$

while with the signature $s_{(j+1) \ldots k\left(-\ell_{2}\right)} s_{\ell_{2}(k+1) \ldots j} s_{\ell_{2}(k+1) \ldots j\left(-\ell_{1}\right)}$, the corresponding CHY-integrand is

$$
T_{j k ; B 32}=T_{j k} \mathcal{P}\left[\ell_{1}, j+1,-\ell_{2}, \ell_{2}\right] \mathcal{P}\left[-\ell_{2}, \ell_{2}, j,-\ell_{1}\right] \mathcal{P}\left[-\ell_{2}, \ell_{2},-\ell_{1}, \ell_{1}\right]
$$


These two are not compatible to each other. They are also not compatible with one-loop tadpole, one-loop massless bubble and two-loop tadpole singularities.

Having the above analysis, now we can write the desired CHY-integrand for ordering $\mathcal{O}_{j k}$ as

$$
\begin{aligned}
\mathcal{I}_{\mathcal{O}_{j k}}^{\mathrm{CHY}}= & T_{j k}-\left(T_{j k ; t_{1}}+T_{j k ; b_{1} j}+T_{j k ; b_{1}(j+1)}+T_{j k ; t_{2}}+T_{j k ; b_{2} k}+T_{j k ; b_{2}(k+1)}\right) \\
& +\left(T_{j k ; t_{1}, t_{2}}+T_{j k ; t_{1}, b_{2} k}+T_{j k ; t_{1}, b_{2}(k+1)}+T_{j k ; b_{1} j, t_{2}}+T_{j k ; b_{1} j, b_{2} k}\right. \\
& +\left(1-\delta_{k, n} \delta_{j, 1}\right) T_{j k ; b_{1} j, b_{2}(k+1)} \\
& \left.+T_{j k ; b_{1}(j+1), t_{2}}+\left(1-\delta_{j+1, k}\right) T_{j k ; b_{1}(j+1), b_{2} k}+T_{j k ; b_{1}(j+1), b_{2}(k+1)}\right) \\
& -\left(\delta_{j+1, k}+\delta_{k, n} \delta_{j, 1}\right)\left(T_{j k ; 2 m 1}+T_{j k ; 2 m 2}\right)-\left(T_{j k ; B 31}+T_{j k ; B 32}\right)
\end{aligned}
$$

where we have inserted $\delta$-functions for special cases $k=(j+1)$ or $k=n, j=1$.

Before ending this subsection, there is a nice feature worth mentioning about one-loop massless bubble singularities. It is well known that integrating the one-loop massless bubble gives zero under some proper regularizations (such as dimensional regularization). We can also see it clearly at the integrand level in the current setup. For the one-loop massless bubble, its integrand is given by $\frac{N(\ell)}{\ell^{2}(\ell-p)^{2}}$. After the partial fraction and momentum shift we get

$$
\frac{N(\ell)}{\ell^{2}(-2 \ell \cdot p)}+\frac{N(\ell)}{(\ell-p)^{2}(2 \ell \cdot p)} \simeq \frac{N(\ell)}{\ell^{2}(-2 \ell \cdot p)}+\frac{N(\ell+p)}{\ell^{2}(2 \ell \cdot p)}
$$

Thus if $N(\ell)=N(\ell+P)$ (which holds for scalar theories), they cancel each other at the integrand level. It is worth emphasizing that the cancelation happens between two different orderings, as already observed in [32], i.e., the orderings $\{\ldots,-\ell, \ell, p, \ldots\}$ and $\{\ldots, p,-\ell, \ell, \ldots\}$. For the two-loop massless bubble, we can do similar manipulation as

$$
\begin{aligned}
& \frac{N\left(\ell_{1}, \ell_{2}\right)}{\ell_{1}^{2}\left(\ell_{1}+p\right)^{2}\left(\ell_{1}-\ell_{2}+p\right)^{2} \ell_{2}^{2}\left(\ell_{2}-p\right)^{2}} \\
& =\frac{N\left(\ell_{1}, \ell_{2}\right)}{\left(\ell_{1}-\ell_{2}+p\right)^{2}}\left(\frac{1}{\ell_{1}^{2}\left(2 \ell_{1} \cdot p\right)}+\frac{1}{\left(\ell_{1}+p\right)^{2}\left(-2 \ell_{1} \cdot p\right)}\right)\left(\frac{1}{\ell_{2}^{2}\left(-2 \ell_{2} \cdot p\right)}+\frac{1}{\left(\ell_{2}-p\right)^{2}\left(2 \ell_{2} \cdot p\right)}\right) \\
& \simeq \frac{N\left(\ell_{1}, \ell_{2}\right)}{\ell_{1}^{2} \ell_{2}^{2}\left(\ell_{1}-\ell_{2}+p\right)^{2}\left(2 \ell_{1} \cdot p\right)\left(-2 \ell_{2} \cdot p\right)}+\frac{N\left(\ell_{1}, \ell_{2}+p\right)}{\ell_{1}^{2} \ell_{2}^{2}\left(\ell_{1}-\ell_{2}\right)^{2}\left(2 \ell_{1} \cdot p\right)\left(2 \ell_{2} \cdot p\right)} \\
& \quad+\frac{N\left(\ell_{1}-p, \ell_{2}\right)}{\ell_{1}^{2} \ell_{2}^{2}\left(\ell_{1}-\ell_{2}\right)^{2}\left(-2 \ell_{1} \cdot p\right)\left(-2 \ell_{2} \cdot p\right)}+\frac{N\left(\ell_{1}-p, \ell_{2}+p\right)}{\ell_{1}^{2} \ell_{2}^{2}\left(\ell_{1}-\ell_{2}-p\right)^{2}\left(-2 \ell_{1} \cdot p\right)\left(2 \ell_{2} \cdot p\right)}
\end{aligned}
$$

Because of the different mixed propagators $\left(\ell_{1}-\ell_{2}+p\right)^{2},\left(\ell_{1}-\ell_{2}-p\right)^{2}$ and $\left(\ell_{1}-\ell_{2}+p\right)^{2}$, we see that even if $N\left(\ell_{1}, \ell_{2}\right)=1$, they do not cancel each other at the integrand level. Because of the explicit cancelation at the integrand level for the one-loop massless bubble after summing over all orderings, we can save the explicit subtraction in (4.39) and simplify it into

$$
\begin{aligned}
\mathcal{I}_{\mathcal{O}_{j k}}^{\mathrm{CHY}}= & \left\{T_{j k}-\left(T_{j k ; t_{1}}+T_{j k ; t_{2}}\right)+T_{j k ; t_{1}, t_{2}}\right\}-\left(\delta_{j+1, k}+\delta_{k, n} \delta_{j, 1}\right)\left(T_{j k ; 2 m 1}+T_{j k ; 2 m 2}\right) \\
& -\left(T_{j k ; B 31}+T_{j k ; B 32}\right) .
\end{aligned}
$$


One can sum the first four terms and simplify it into

$$
T_{j k}\left(1-\frac{z_{j\left(-\ell_{1}\right)} z_{\ell_{1}(j+1)}}{z_{j \ell_{1}} z_{\left(-\ell_{1}\right)(j+1)}}\right)\left(1-\frac{z_{k\left(-\ell_{2}\right)} z_{\ell_{2}(k+1)}}{z_{k \ell_{2}} z_{\left(-\ell_{2}\right)(k+1)}}\right)=T_{j k} \frac{z_{j(j+1)} z_{\left(-\ell_{1}\right) \ell_{1}}}{z_{j \ell_{1}} z_{\left(-\ell_{1}\right)(j+1)}} \frac{z_{k(k+1)} z_{\left(-\ell_{2}\right) \ell_{2}}}{z_{k \ell_{2}} z_{\left(-\ell_{2}\right)(k+1)}} .
$$

Although one can continue to add later four terms, (4.42) has a clearer physical picture.

\subsubsection{The CHY-integrand for ordering $\mathcal{O}_{j}$}

Having done the ordering $\mathcal{O}_{j k}$, we now consider the ordering $\mathcal{O}_{j}$. In this case the full tree-level amplitude of $(n+4)$-legs is given by the following CHY-integrand

$$
T_{j}=\frac{1}{z_{12}^{2} \ldots z_{j\left(-\ell_{1}\right)}^{2} z_{\left(-\ell_{1}\right) \ell_{1}}^{2} z_{\ell_{1}\left(-\ell_{2}\right)}^{2} z_{\left(-\ell_{2}\right) \ell_{2}}^{2} z_{\ell_{2}(j+1)}^{2} \cdots z_{n 1}^{2}}
$$

Now we consider various forward limits, which can be produced in this ordering by checking figure 5, figure 6 and figure 7 :

- Firstly there are one-loop tadpole singularities, thus we have the corresponding CHYintegrands

$$
T_{j ; t_{1}}=T_{j} \mathcal{P}\left[j,-\ell_{1}, \ell_{1},-\ell_{2}\right], \quad T_{j ; t_{2}}=T_{j} \mathcal{P}\left[\ell_{1},-\ell_{2}, \ell_{2}, j+1\right]
$$

for $\ell_{1}$-tadpole and $\ell_{2}$-tadpole respectively.

- Secondly, there are one-loop massless bubbles. With the $j$-th leg, there are bubbles with poles $s_{j\left(-\ell_{1}\right)} s_{j\left(-\ell_{1}\right) \ell_{1}}$ and the corresponding CHY-integrand is

$$
T_{j ; b_{1} j}=T_{j} \mathcal{P}\left[j-1, j,-\ell_{1}, \ell_{1}\right] \mathcal{P}\left[j-1, j, \ell_{1},-\ell_{2}\right] .
$$

With the $(j+1)$-th leg, there are bubbles with poles $s_{\ell_{2}(j+1)} s_{\left(-\ell_{2}\right) \ell_{2}(j+1)}$ and the corresponding CHY-integrand is

$$
T_{j ; b_{2}(j+1)}=T_{j} \mathcal{P}\left[-\ell_{2}, \ell_{2}, j+1, j+2\right] \mathcal{P}\left[\ell_{1},-\ell_{2}, j+1, j+2\right] .
$$

- Again, because of the compatibility we have the following four combinations from $\ell_{1}$ and $\ell_{2}$ one-loop forward singularities:

$$
\begin{aligned}
T_{j ; t_{1}, t_{2}}= & T_{j} \mathcal{P}\left[j,-\ell_{1}, \ell_{1},-\ell_{2}\right] \mathcal{P}\left[\ell_{1},-\ell_{2}, \ell_{2}, j+1\right] \\
T_{j ; t_{1}, b_{2}(j+1)=} & T_{j} \mathcal{P}\left[j,-\ell_{1}, \ell_{1},-\ell_{2}\right] \mathcal{P}\left[-\ell_{2}, \ell_{2}, j+1, j+2\right] \mathcal{P}\left[\ell_{1},-\ell_{2}, j+1, j+2\right], \\
T_{j ; b_{1} j, t_{2}}= & T_{j} \mathcal{P}\left[j-1, j,-\ell_{1}, \ell_{1}\right] \mathcal{P}\left[j-1, j, \ell_{1},-\ell_{2}\right] \mathcal{P}\left[\ell_{1},-\ell_{2}, \ell_{2}, j+1\right] \\
T_{j ; b_{1} j, b_{2}(j+1)=} & T_{j} \mathcal{P}\left[j-1, j,-\ell_{1}, \ell_{1}\right] \mathcal{P}\left[j-1, j, \ell_{1},-\ell_{2}\right] \mathcal{P}\left[-\ell_{2}, \ell_{2}, j+1, j+2\right] \\
& \mathcal{P}\left[\ell_{1},-\ell_{2}, j+1, j+2\right]
\end{aligned}
$$

- Now we discuss two-loop massless bubbles. Again let us start with two-loop bubble topologies. From figure 6 we can see that beside the pole $s_{\ell_{1}\left(-\ell_{2}\right)}$, there is a free parameter $k$ with $k=(j+1),(j+2), \ldots, n, 1, \ldots, j-1$. For each $k$, there is one 
bubble structure with pole $s_{\ell_{1}\left(-\ell_{2}\right)} s_{(j+1) \ldots k} s_{\ell_{2}(j+1) \ldots k} s_{(k+1) \ldots j} s_{(k+1) \ldots j\left(-\ell_{1}\right)}$ and the corresponding $\mathrm{CHY}$-integrand is given by

$$
\begin{aligned}
T_{j ; 2 m}[k]= & T_{j} \mathcal{P}\left[-\ell_{1}, \ell_{1},-\ell_{2}, \ell_{2}\right] \mathcal{P}\left[\ell_{2}, j+1, k, k+1\right] \mathcal{P}\left[-\ell_{2}, \ell_{2}, k, k+1\right] \\
& \mathcal{P}\left[k, k+1, j,-\ell_{1}\right] \mathcal{P}\left[k, k+1,-\ell_{1}, \ell_{1}\right] .
\end{aligned}
$$

Again, for general $k$, they are massive bubbles. Only when $k=j+1$ or $k=j-1$ will we get the massless bubbles. The CHY-integrands for both special cases can be trivially obtained from (4.52) by setting $k=j+1$ and $k=j-1$. For $k=j+1$, we have $\mathcal{P}\left[\ell_{2}, j+1, k, k+1\right]=1$ while for $k=j-1$, we have $\mathcal{P}\left[k, k+1, j,-\ell_{1}\right]=1$.

- The last piece we need to exclude is the (B-3) part in figure 4. From figure 7, we see that with the pole $s_{\ell_{1}\left(-\ell_{2}\right)} s_{\ell_{2}(j+1) \ldots j}$, the CHY-integrand is given by

$$
T_{j ; B 31}=T_{j} \mathcal{P}\left[-\ell_{1}, \ell_{1},-\ell_{2}, \ell_{2}\right] \mathcal{P}\left[-\ell_{2}, \ell_{2}, j,-\ell_{1}\right],
$$

while with the pole $s_{\ell_{1}\left(-\ell_{2}\right)} s(j+1) \ldots j\left(-\ell_{1}\right)$, the CHY-integrand is given by

$$
T_{j ; B 32}=T_{j} \mathcal{P}\left[-\ell_{1}, \ell_{1},-\ell_{2}, \ell_{2}\right] \mathcal{P}\left[\ell_{2}, j+1,-\ell_{1}, \ell_{1}\right] .
$$

With the above analysis, we can write the CHY-integrand for ordering $\mathcal{O}_{j}$ as

$$
\begin{aligned}
\mathcal{I}_{\mathcal{O}_{j}}^{\mathrm{CHY}}= & T_{j}-\left(T_{j ; t_{1}}+T_{j ; t_{2}}+T_{j ; b_{1} j}+T_{j ; b_{2}(j+1)}\right) \\
& +\left(T_{j ; t_{1}, t_{2}}+T_{j ; t_{1}, b_{2}(j+1)}+T_{j ; b_{1} j, t_{2}}+T_{j ; b_{1} j, b_{2}(j+1)}\right) \\
& -T_{j ; 2 m}[j+1]-T_{j ; 2 m}[j-1]-\left(T_{j ; B 31}+T_{j ; B 32}\right)
\end{aligned}
$$

Again we can neglect one-loop massless bubbles in order to simplify the expression, although we prefer the more complicated one (4.55) to have a clearer physical picture.

\subsection{The CHY-construction of reducible two-loop diagrams}

As mentioned in subsection 4.2, for two-loop diagrams, there are special case (called the reducible two-loop diagrams), which will make the partial fraction problematic. After a careful analysis, we have reduced the problem to the one-loop case in (4.12) and (4.13). Although as we have mentioned, we will treat this part as the known data, in this subsection, we will try to give a direct CHY-construction of these reducible two-loop diagrams at two-loop level.

Let us recall the general expressions for reducible two-loop diagrams. From diagram (a) of figure 9 we can read off

$$
\frac{1}{\ell_{1}^{2}\left(\prod_{i=1}^{m}\left(\ell_{1}+P_{i}\right)\right) \ell_{1}^{2}\left(\ell_{1}-\ell_{2}\right)^{2} \ell_{2}^{2}}
$$

with the chosen loop momenta. Now from these $n$ external momenta $k_{i}$ satisfying $\sum_{i=1}^{n} k_{i}=$ 0 , we try to construct $(n+1)$ massless momenta by the following way. Picking, for example, $k_{n}$ and a massless momentum $k_{s}$ such that $k_{n} \cdot k_{s}=0$, then the $(n+1)$ massless momenta 


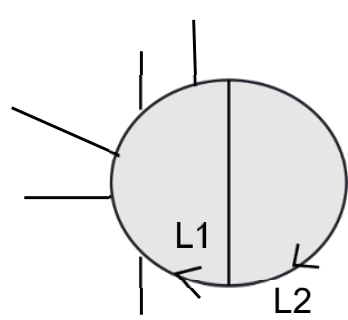

(a)

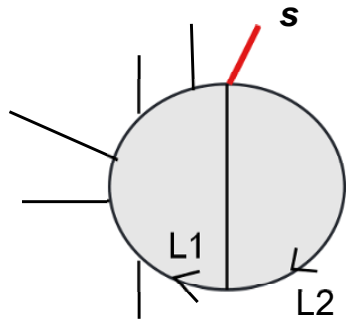

(b)

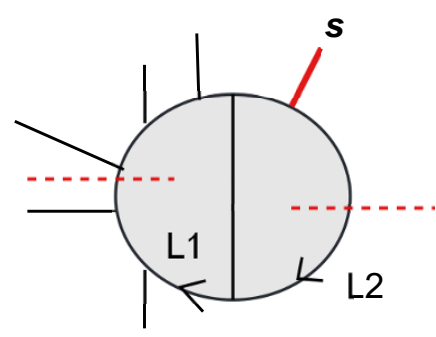

(c)

Figure 9. (a) The reducible two-loop diagrams; (b) After adding a particle to the vertex. Then owever this vertex has four legs. (c) Moving the added particle to $\ell_{2}$-loop to make the vertex cubic. Furthermore, we have illustrated possible cuts for this cubic diagram.

can be arranged to be $\left\{k_{1}, \ldots, k_{n-1}, k_{n}-t k_{s}, t k_{s}\right\}$. Using this construction, each diagram (a) in figure 9 will have a corresponding diagram (b) in figure 9 with the expression

$$
\frac{1}{\ell_{1}^{2}\left(\prod_{i=1}^{m}\left(\ell_{1}+P_{i}\right)^{2}\right)\left(\ell_{1}-t k_{s}\right)^{2}\left(\ell_{1}-\ell_{2}\right)^{2} \ell_{2}^{2}}
$$

It is easy to see that under the soft limit $t \rightarrow 0,(4.57)$ reduces to (4.56). Although it looks satisfactory, it is no longer the $\phi^{3}$ theory since we now have one vertex with four legs. We can remedy this by moving the leg $s$ to the $\ell_{2}$-loop as what we did in diagram (c) in figure 9. Thus (4.57) can be written as

$$
\left(\ell_{2}-t k_{s}\right)^{2} \times \frac{1}{\ell_{1}^{2}\left(\prod_{i=1}^{m}\left(\ell_{1}+P_{i}\right)^{2}\right)\left(\ell_{1}-t k_{s}\right)^{2}\left(\ell_{1}-\ell_{2}\right)^{2} \ell_{2}^{2}\left(\ell_{2}-t k_{s}\right)^{2}}
$$

Now formula (4.58) can have the CHY-construction by the standard procedure, i.e., the partial fraction and momentum shift, thus we get

$$
\frac{-2 t \widetilde{\ell}_{2} \cdot k_{s}}{\ell_{1}^{2} \ell_{2}^{2}} \mathcal{A}\left( \pm \widetilde{\ell}_{1}, \pm \widetilde{\ell}_{2}, 1, \ldots, k_{n}-t k_{s}, k_{s}\right)
$$

where $\mathcal{A}$ is a certain tree-level amplitude with $(n+5)$-points, where $\widetilde{\ell}_{1}, \widetilde{\ell}_{2}$ are on-shell momenta in higher dimensions.

Having the above picture, now we can present the explicit CHY-construction for the term $\frac{1}{\ell_{1}^{2}\left(\ell_{1}-\ell_{2}\right)^{2} \ell_{2}^{2}} \times \mathcal{I}^{1-\text { loop }}\left(1,2, \ldots, n, \ell_{1}\right)$ in (4.13) as the soft limit of (4.59) (other terms in (4.13) can be obtained by cyclic permutations). Here $\mathcal{A}$ is given by the sum of the following orderings of trees:

$$
\mathcal{O}_{j}=\left\{\left(-\ell_{2}, \ell_{2}\right), 1, \ldots, j,\left(-\ell_{1}, \ell_{1}\right), j+1, \ldots, n, s\right\}, \quad j=0,1, \ldots, n
$$

where $j=0$ means the pair $\left(-\ell_{1}, \ell_{1}\right)$ is inserted in front of the leg 1 . For the $j$-th ordering $(j \neq 0, n)$, the signature of pole is $s_{s\left(-\ell_{2}\right)} s_{\ell_{1}(j+1) \ldots n} s_{\ell_{2} 1 \ldots j\left(-\ell_{1}\right)} s_{1 \ldots j\left(-\ell_{1}\right)}$, thus the CHYintegrand is given by

$$
T_{j}=\mathcal{I}_{j} \mathcal{P}\left[n, s,-\ell_{2}, \ell_{2}\right] \mathcal{P}\left[-\ell_{1}, \ell_{1}, n, s\right] \mathcal{P}\left[-\ell_{2}, \ell_{2},-\ell_{1}, \ell_{1}\right] \mathcal{P}\left[\ell_{2}, 1,-\ell_{1}, \ell_{1}\right]
$$


with

$$
\mathcal{I}_{j}=\frac{1}{z_{\left(-\ell_{2}\right) \ell_{2}}^{2} z_{\ell_{2} 1}^{2} \ldots z_{j\left(-\ell_{1}\right)}^{2} z_{\left(-\ell_{1}\right) \ell_{1}}^{2} z_{\ell_{1}(j+1)}^{2} \ldots z_{n s}^{2} z_{s\left(-\ell_{2}\right)}^{2}} .
$$

For $j=0$, the signature of pole is $s_{s\left(-\ell_{2}\right)} s_{\ell_{1} 1 \ldots n} s_{\ell_{2}\left(-\ell_{1}\right)}$, thus the CHY-integrand is given by

$$
T_{j=0}=\mathcal{I}_{j=0} \mathcal{P}\left[n, s,-\ell_{2}, \ell_{2}\right] \mathcal{P}\left[-\ell_{1}, \ell_{1}, n, s\right] \mathcal{P}\left[-\ell_{2}, \ell_{2},-\ell_{1}, \ell_{1}\right]
$$

For $j=n$, the signature of pole is $s_{s\left(-\ell_{2}\right)} s_{\ell_{1} s\left(-\ell_{2}\right)} s_{1 \ldots n\left(-\ell_{1}\right)}$, thus the CHY-integrand is given by

$$
T_{j=n}=\mathcal{I}_{j=n} \mathcal{P}\left[\ell_{1}, s,-\ell_{2}, \ell_{2}\right] \mathcal{P}\left[-\ell_{1}, \ell_{1},-\ell_{2}, \ell_{2}\right] \mathcal{P}\left[\ell_{2}, 1,-\ell_{1}, \ell_{1}\right]
$$

Next we need to subtract forward singularities associated to the above orderings:

- The tadpole structure could appear when $j=0$ or $j=n$. For $j=0$ the signature will be $s_{s\left(-\ell_{2}\right)} s_{\ell_{1} 1 \ldots n} s_{\ell_{2}\left(-\ell_{1}\right)}$ multiplied by an extra factor $s_{1 \ldots n}$, thus we have

$$
T_{j=0 ; t}=T_{j=0} \mathcal{P}\left[\ell_{1}, 1, n, s\right] .
$$

For $j=n$ the signature will be $s_{s\left(-\ell_{2}\right)} s_{\ell_{1} s\left(-\ell_{2}\right)} s_{1 \ldots n\left(-\ell_{1}\right)}$ multiplied by an extra factor $s_{1 \ldots n}$, thus we have

$$
T_{j=n ; t}=T_{j=n} \mathcal{P}\left[\ell_{2}, 1, n,-\ell_{1}\right] .
$$

- The massless bubble structure could appear when $j=0,1, n-1, n$. For $j=0$ the signature will be $s_{s\left(-\ell_{2}\right)} s_{\ell_{1} 1 \ldots n} s_{\ell_{2}\left(-\ell_{1}\right)}$ multiplied by either factor $s_{\ell_{1} 1} s_{2 \ldots n}$ or $s_{1 \ldots(n-1)} s_{\ell_{1} 1 \ldots(n-1)}$, thus the corresponding CHY-integrands are

$$
\begin{aligned}
& T_{j=0 ; 61}=T_{j=0} \mathcal{P}\left[-\ell_{1}, \ell_{1}, 1,2\right] \mathcal{P}[1,2, n, s], \\
& T_{j=0 ; 62}=T_{j=0} \mathcal{P}\left[-\ell_{1}, \ell_{1}, n-1, n\right] \mathcal{P}\left[\ell_{1}, 1, n-1, n\right] .
\end{aligned}
$$

For $j=n$ the signature will be $s_{s\left(-\ell_{2}\right)} s_{\ell_{1} s\left(-\ell_{2}\right)} s_{1 \ldots n\left(-\ell_{1}\right)}$ multiplied by either factor $s_{\left(-\ell_{1}\right) n} s_{1 \ldots(n-1)}$ or $s_{2 \ldots n} s_{2 \ldots n\left(-\ell_{1}\right)}$, thus we have

$$
\begin{aligned}
& T_{j=n ; b 1}=T_{j=n} \mathcal{P}\left[n-1, n,-\ell_{1}, \ell_{1}\right] \mathcal{P}\left[\ell_{2}, 1, n-1, n\right] \\
& T_{j=n ; b 2}=T_{j=n} \mathcal{P}\left[1,2, n,-\ell_{1}\right] \mathcal{P}\left[1,2,-\ell_{1}, \ell_{1}\right] .
\end{aligned}
$$

For $j=1$, the signature will be the one of $T_{j=1}$ multiplied by an extra factor $s_{2 \ldots n}$, thus the CHY-integrand is

$$
T_{j=1 ; b}=T_{j=1} \mathcal{P}\left[\ell_{1}, 2, n, s\right] .
$$

For $j=n-1$, the signature will be the one of $T_{j=n-1}$ multiplied by an extra factor $s_{1 \ldots(n-1)}$, thus the CHY-integrand is

$$
T_{j=n-1 ; b}=T_{j=n-1} \mathcal{P}\left[\ell_{2}, 1, n-1,-\ell_{1}\right] .
$$


Putting all of them together, we finally get the following CHY-integrand:

$$
\begin{aligned}
\mathcal{I}_{\mathcal{A}}(1,2 \ldots, n)= & \sum_{j=0}^{n} T_{j}-\left(T_{j=0 ; t}+T_{j=n ; t}\right)-\left(T_{j=0 ; b 1}+T_{j=0 ; b 2}+T_{j=n ; b 1} T_{j=n ; n 2}\right) \\
& -\left(T_{j=1 ; b}+T_{j=n-1 ; b}\right)
\end{aligned}
$$

Before ending this subsection, we would like to remark that although we have presented a solution using the soft limit, a more direct treatment is yet desired, but now we need to understand how to construct CHY-integrands with double poles at tree-level. This will be an interesting topic to investigate.

\section{Counting}

Having reduced the two-loop problem to the tree level (i.e., the loop scattering equations and CHY-integrands) using the dimensional reduction, the checking to check the proposal for the two-loop case is now to check the corresponding tree one. Since the latter has been extensively checked both numerically and analytically (especially the powerful mapping rule), our proposal should be correct. In this section, we will give a further evidence to support this claim by comparing the numbers of terms produced by Feynman diagrams directly or by the CHY-formula. From now on, contributions from reducible two-loop diagrams will be excluded.

\subsection{Counting from CHY-formula}

Since the entire result is obtained by summing over $2 n$ orderings of type $\mathcal{O}_{j}$ and $n(n-1)$ orderings of type $\mathcal{O}_{j k}$, we will count terms from these two types one by one.

The type $\mathcal{O}_{j}$. Let us start with the formula (4.55). The first term gives the full tree-level amplitude of $(n+4)$-points, so it contains $C(n+4)$ terms. For $T_{j ; t_{1}}$, since all tree diagrams have the pole $s_{\left(-\ell_{1}\right) \ell_{2}}$, these two legs have been effectively grouped as a single leg, thus all of them become the tree-level diagrams of the $(n+3)$-points, so it contains $C(n+3)$ terms. For $T_{j ; B 31}$ and $T_{j ; B 32}$, we see that they are effectively tree amplitudes of $(n+2)$-points. Similar arguments give

$$
\begin{array}{rlrl}
\mathcal{N}\left[T_{j}\right] & =C(n+4), & \mathcal{N}\left[T_{j ; t_{1}}\right] & =\mathcal{N}\left[T_{j ; t_{2}}\right]=C(n+3), \\
\mathcal{N}\left[T_{j ; b_{1} j}\right] & =\mathcal{N}\left[T_{j ; b_{2}(j+1)}\right]=C(n+2), \\
\mathcal{N}\left[T_{j ; t_{1}, t_{2}}\right] & =C(n+2), & \mathcal{N}\left[T_{j ; t_{1}, b_{2}(j+1)}\right] & =\mathcal{N}\left[T_{j ; b_{1} j, t_{2}}\right]=C(n+1), \\
\mathcal{N}\left[T_{j ; b_{1} j, b_{2}(j+1)}\right] & =C(n), & \mathcal{N}\left[T_{j ; B 31}\right] & =\mathcal{N}\left[T_{j ; B 32}\right]=C(n+2)
\end{array}
$$

For general $T_{j ; 2 m}[k]$, the counting is a bit more complicated. For a given $k$, we have two tree diagrams: one with $2+(k-j)$ legs and one with $n-(k-j)+2$ legs. Furthermore, external legs in each group will merge before meeting $\ell_{i}$ (i.e., to give the tree-structure of $(1+(k-j))$ points and $(n-(k-j)+1)$-points $)$, thus we will count $C(k-j+1) C(n-(k-j)+1)$ terms. However, for massless bubbles, we only need to consider the case $k-j=1$ or $n-(k-j)=1$ and both cases contain $C(n)$ terms. 
Putting all of them together, we finally get

$$
\mathcal{N}\left[\mathcal{O}_{j}\right]=C(n+4)-2 C(n+3)-3 C(n+2)+2 C(n+1)-C(n) .
$$

This expression is independent of $j$ as it should. There are $2 n$ terms of this type, so the final number of terms of this type should be

$$
\mathcal{N}_{I}=2 n\{C(n+4)-2 C(n+3)-3 C(n+2)+2 C(n+1)-C(n)\} .
$$

The type $\mathcal{O}_{j k}$. For this type, we start with (4.39) where $1 \leq j<k \leq n$. Using similar arguments we figure out the counting for each case:

$$
\begin{aligned}
\mathcal{N}\left[T_{j k}\right] & =C(n+4), \quad \mathcal{N}\left[T_{j k ; t_{1}}\right]=\mathcal{N}\left[T_{j k ; t_{2}}\right]=C(n+3), \quad \mathcal{N}\left[T_{j k ; t_{1}, t_{2}}\right]=C(n+2), \\
\mathcal{N}\left[T_{j k ; b_{1}}\right] & =\mathcal{N}\left[T_{j k ; b_{1}(j+1)}\right]=\mathcal{N}\left[T_{j k ; b_{2} k}\right]=\mathcal{N}\left[T_{j k ; b_{2}(k+1)}\right]=C(n+2), \\
\mathcal{N}\left[T_{j k ; t_{1}, b_{2} k}\right] & =\mathcal{N}\left[T_{j k ; t_{1}, b_{2}(k+1)}\right]=\mathcal{N}\left[T_{j k ; b_{1}(j+1), t_{2}}\right]=\mathcal{N}\left[T_{j k ; b_{1} j, b_{2} k}\right]=C(n+1), \\
\mathcal{N}\left[T_{j k ; b_{1} j, b_{2} k}\right] & =\mathcal{N}\left[T_{j k ; b_{1}(j+1), b_{2}(k+1)}\right]=\mathcal{N}\left[T_{j k ; b_{1}(j+1), b_{2} k}\right]=\mathcal{N}\left[T_{j k ; b_{1}(j+1), b_{2}(k+1)}\right]=C(n), \\
\mathcal{N}\left[T_{j k ; 2 m 1}\right] & =\mathcal{N}\left[T_{j k ; 2 m 2}\right]=C(k-j+1) C(n-(k-j)+1), \\
\mathcal{N}\left[T_{j k ; B 31}\right] & =\mathcal{N}\left[T_{j k ; B 32}\right]=C(k-j+2) C(n-(k-j)+2) .
\end{aligned}
$$

Thus from (4.39) we get

$$
\begin{aligned}
\mathcal{N}\left[\mathcal{Q}_{j k}\right]= & C(n+4)-2 C(n+3)-3 C(n+2)+4 C(n+1)+\left(4-\delta_{j+1, k}-\delta_{k, n} \delta_{j, 1}\right) C(n) \\
& -2\left(\delta_{j+1, k}+\delta_{k, n} \delta_{j, 1}\right) C(k-j+1) C(n-(k-j)+1) \\
& -2 C(k-j+2) C(n-(k-j)+2)
\end{aligned}
$$

for general choice of $j, k$. Thus when $k=j+1$ or $k=n, j=1$, the counting gives

$$
\begin{aligned}
\mathcal{N}^{s} & =C(n+4)-2 C(n+3)-3 C(n+2)+4 C(n+1)+3 C(n)-2 C(n)-2 C(n+1) \\
& =C(n+4)-2 C(n+3)-3 C(n+2)+2 C(n+1)+C(n) .
\end{aligned}
$$

For other cases, we have

$$
\begin{aligned}
\mathcal{N}^{g}\left[\mathcal{Q}_{j k}\right]= & C(n+4)-2 C(n+3)-3 C(n+2)+4 C(n+1)+4 C(n) \\
& -2 C(k-j+2) C(n-(k-j)+2) .
\end{aligned}
$$

Now we sum over all $(j, k)$ pairs. For the special cases, there are $2 n$ terms, so we have

$$
\mathcal{N}_{I I, A}=2 n \mathcal{N}^{s}=2 n\{C(n+4)-2 C(n+3)-3 C(n+2)+2 C(n+1)+C(n)\} .
$$

For other $(n(n-1)-2 n)$ cases, we get the sum

$$
\mathcal{N}_{I I, B}=2\left\{\sum_{k=3}^{n-1} \mathcal{N}^{g}\left[\mathcal{Q}_{j=1, k}\right]+\sum_{j=2}^{n-2} \sum_{k=j+2}^{n} \mathcal{N}^{g}\left[\mathcal{Q}_{j k}\right]\right\} .
$$

Putting all together, we find that the total number of terms given by the CHYformula is

$$
\mathcal{N}_{\mathrm{CHY}}=\mathcal{N}_{I}+\mathcal{N}_{I I, A}+\mathcal{N}_{I I, B} .
$$




\subsection{Counting from Feynman diagrams}

Now we will do the counting using Feynman diagrams given in figure 4 directly. Although we will count terms for Type (A) and Type (B) separately, they do share the same one-loop building block as indicated by the red square in figure 4 (the $n_{L}$ part of Type A), thus we need to consider contributions from the building block first. To deal with this, it is crucial to recall that after applying the partial fraction to expression $\frac{1}{\Pi\left(\ell+K_{i}\right)^{2}}$, we will get terms of the form $\frac{1}{\left(\ell_{1}+K_{i}\right)^{2}} \times F_{i}$ for each $K_{i}$. Now we count terms with the same propagator $\frac{1}{\left(\ell_{1}+K_{i}\right)^{2}}$. Since the partial fraction has the physical picture as cutting the propagator to make it on-shell, the building block has been separated into two trees. One has $n_{1}$ external legs in the lower part (so the entire structure is a tree of $\left(n_{1}+2\right)$-points), while another one has $n_{2}=n_{L}-n_{1}$ external legs in the upper part (so the entire structure is a tree of $\left(n_{2}+2\right)$-points). Using the formula (2.5) we get the number of terms associated to this propagator, which is $C\left(n_{1}+2\right) C\left(n-n_{1}+2\right)$. Summing over all splitting, we get the number of terms for the one-loop building block as

$$
\mathcal{B}(n)=\sum_{n_{1}=0}^{n} C\left(n_{1}+2\right) C\left(n-n_{1}+2\right) .
$$

Having the building block, we can count terms for the two types of diagrams in figure 4 . For Type (A), since we require $n_{L}, n_{R} \geq 2$ to avoid one-loop tadpoles and massless bubbles, the total number of terms is given by

$$
\mathcal{N}_{F ; A}(n)=n \sum_{n_{L}=2}^{n-2} \sum_{n_{R}=2}^{n-n_{L}} \mathcal{B}\left(n_{L}\right) \mathcal{B}\left(n_{R}\right) C\left(n-n_{L}-n_{R}+2\right)\left(n-n_{L}-n_{R}+1\right) .
$$

Let us give a brief explanation of formula (5.12). Firstly the factor $n$ comes from the sum over all cyclic orderings. The cyclic sum also symmetrizes the two loop momenta $\ell_{1}, \ell_{2}$ in the integrand. Secondly, the sum is over all possible distributions of $n$ legs separated into four subsets $n_{L}, m_{u}, m_{d}, n_{R}$ with $n_{L} \geq 2, n_{R} \geq 2$ and $m_{u}, m_{d} \geq 0$. Thirdly, from the Feynman diagrams, it can be seen that the middle part is just the tree amplitude of $\left(2+m_{u}+m_{d}\right)=\left(n-n_{L}-n_{R}+2\right)$-points. Furthermore, there are $\left(n-n_{L}-n_{R}+1\right)$ ways to distribute $m_{u}, m_{d}$ given $n_{L}, n_{R}$, so the contribution from the middle part is counted by $C\left(n-n_{L}-n_{R}+2\right)\left(n-n_{L}-n_{R}+1\right)$.

For Type (B), the counting is much simpler. Using the formula for the building block, we get

$$
\mathcal{N}_{F ; B}(n)=n \sum_{n_{L}=1}^{n-1} \mathcal{B}\left(n_{L}\right) \mathcal{B}\left(n-n_{L}\right)-2^{3} n C(n)
$$

Now let us explain formula (5.13). Firstly the factor $n$ comes again from the sum over cyclic orderings. Secondly, to exclude reducible two-loop diagrams, we require $n_{L} \geq 1, n_{R} \geq 1$ when summing over all different distributions for $n$ to $n_{L}$ and $n_{R}$. Furthermore, There are two special cases corresponding to two-loop massless bubbles. One is $n_{L}=1$ and another is $n_{R}=1$. They are multiplied by $C(n)$ because the remaining $(n-1)$-legs must be grouped 
together as one. The factor $2^{3}$ is due to the fact that each massless bubble will produce four trees by different combinations of two cuts, plus that there are two choices $n_{L}=1$ or $n_{R}=1$.

Summing these two parts, finally we get the number of terms after the partial fraction of the expressions from Feynman diagrams

$$
\mathcal{N}_{F}(n)=\mathcal{N}_{F ; B}(n)+\mathcal{N}_{F ; A}(n)
$$

It can be checked that (5.14) equals to (5.10) although they are completely different expressions. This matching serves as a strong consistency check.

\section{Conclusion}

In this paper, we have established the all-loop scattering equations by lifting the loop momenta to higher dimensions. From this new perspective, we have effectively reduced the loop problem to the forward limit of the corresponding tree one. One technical difficulty of this construction is how to remove forward singularities of the corresponding tree parts. Applying it to the bi-adjoint $\phi^{3}$ theory, we have demonstrated how to achieve this goal for two-loop planar integrands. The method is based on a nice understanding of the mapping rule, especially how to construct the CHY-integrand which produces tree amplitudes with a fixed pole structure. We have confirmed our two-loop results of $\phi^{3}$ theory by matching the number of terms obtained by two different methods.

Although we have focused on the planar part only in this paper, we the same idea should work for the non-planar part as well as non-color-ordered loop amplitudes. We believe that this construction can be generalized to higher loops, at least for $\phi^{3}$ theory. Another important issue is to understand how to remove the forward singularities of YangMills theory and gravity based on these results.

\section{Acknowledgments}

We would like to thank the early participation of Christian Baadsgaard, N. E. J. BjerrumBohr, Jacob L. Bourjaily, Simon Caron-Huot and Poul H. Damgaard. We would thank discussions with Song He, Rijun Huang, Qinjun jin, Minxin Luo, Junjie Rao and suggestions for this draft by N. E. J. Bjerrum-Bohr, Poul H. Damgaard and Rijun Huang. And we would like to thank the hospitality of Niels Bohr International Academy where this project was initiated. Finally we thank Junjie Rao for proofreading of the paper. This work is supported by Qiu-Shi Funding and the Chinese NSF funding under contracts No.11135006, No.11125523 and No.11575156.

Open Access. This article is distributed under the terms of the Creative Commons Attribution License (CC-BY 4.0), which permits any use, distribution and reproduction in any medium, provided the original author(s) and source are credited. 


\section{References}

[1] F. Cachazo, S. He and E.Y. Yuan, Scattering equations and Kawai-Lewellen-Tye orthogonality, Phys. Rev. D 90 (2014) 065001 [arXiv:1306.6575] [INSPIRE].

[2] F. Cachazo, S. He and E.Y. Yuan, Scattering of Massless Particles in Arbitrary Dimensions, Phys. Rev. Lett. 113 (2014) 171601 [arXiv:1307.2199] [InSPIRE].

[3] F. Cachazo, S. He and E.Y. Yuan, Scattering of Massless Particles: Scalars, Gluons and Gravitons, JHEP 07 (2014) 033 [arXiv: 1309.0885] [INSPIRE].

[4] F. Cachazo, S. He and E.Y. Yuan, Einstein-Yang-Mills Scattering Amplitudes From Scattering Equations, JHEP 01 (2015) 121 [arXiv: 1409.8256] [INSPIRE].

[5] F. Cachazo, S. He and E.Y. Yuan, Scattering Equations and Matrices: From Einstein To Yang-Mills, DBI and NLSM, JHEP 07 (2015) 149 [arXiv:1412.3479] [INSPIRE].

[6] D. Fairlie and D. Roberts, Dual Models without Tachyons - a New Approach, unpublished, Durham preprint PRINT-72-2440 (1972).

[7] D. Roberts, Mathematical Structure of Dual Amplitudes, Ph.D. Thesis, Durham University, Durham U.K. (1972).

[8] D.B. Fairlie, A Coding of Real Null Four-Momenta into World-Sheet Co-ordinates, Adv. Math. Phys. 2009 (2009) 284689 [arXiv:0805. 2263] [inSPIRE].

[9] D.J. Gross and P.F. Mende, String Theory Beyond the Planck Scale, Nucl. Phys. B 303 (1988) 407 [inSPIRE].

[10] E. Witten, Parity invariance for strings in twistor space, Adv. Theor. Math. Phys. 8 (2004) 779 [hep-th/0403199] [INSPIRE].

[11] P. Caputa and S. Hirano, Observations on Open and Closed String Scattering Amplitudes at High Energies, JHEP 02 (2012) 111 [arXiv:1108.2381] [INSPIRE].

[12] P. Caputa, Lightlike contours with fermions, Phys. Lett. B 716 (2012) 475 [arXiv: 1205.6369] [INSPIRE].

[13] Y. Makeenko and P. Olesen, The QCD scattering amplitude from area behaved Wilson loops, Phys. Lett. B 709 (2012) 285 [arXiv:1111.5606] [INSPIRE].

[14] F. Cachazo, Fundamental BCJ Relation in $N=4$ SYM From The Connected Formulation, arXiv: 1206.5970 [INSPIRE].

[15] L. Dolan and P. Goddard, Proof of the Formula of Cachazo, He and Yuan for Yang-Mills Tree Amplitudes in Arbitrary Dimension, JHEP 05 (2014) 010 [arXiv:1311.5200] [INSPIRE].

[16] R. Britto, F. Cachazo and B. Feng, New recursion relations for tree amplitudes of gluons, Nucl. Phys. B 715 (2005) 499 [hep-th/0412308] [INSPIRE].

[17] R. Britto, F. Cachazo, B. Feng and E. Witten, Direct proof of tree-level recursion relation in Yang-Mills theory, Phys. Rev. Lett. 94 (2005) 181602 [hep-th/0501052] [InSPIRE].

[18] L. Mason and D. Skinner, Ambitwistor strings and the scattering equations, JHEP 07 (2014) 048 [arXiv: 1311.2564] [INSPIRE].

[19] N. Berkovits, Infinite Tension Limit of the Pure Spinor Superstring, JHEP 03 (2014) 017 [arXiv: 1311.4156] [INSPIRE]. 
[20] H. Gomez and E.Y. Yuan, N-point tree-level scattering amplitude in the new Berkovits" string, JHEP 04 (2014) 046 [arXiv: 1312.5485] [INSPIRE].

[21] T. Adamo, E. Casali and D. Skinner, Ambitwistor strings and the scattering equations at one loop, JHEP 04 (2014) 104 [arXiv:1312.3828] [INSPIRE].

[22] Y. Geyer, A.E. Lipstein and L.J. Mason, Ambitwistor Strings in Four Dimensions, Phys. Rev. Lett. 113 (2014) 081602 [arXiv:1404.6219] [inSPIRE].

[23] Y. Geyer, A.E. Lipstein and L. Mason, Ambitwistor strings at null infinity and (subleading) soft limits, Class. Quant. Grav. 32 (2015) 055003 [arXiv:1406.1462] [INSPIRE].

[24] E. Casali and P. Tourkine, Infrared behaviour of the one-loop scattering equations and supergravity integrands, JHEP 04 (2015) 013 [arXiv:1412.3787] [INSPIRE].

[25] T. Adamo and E. Casali, Scattering equations, supergravity integrands and pure spinors, JHEP 05 (2015) 120 [arXiv: 1502.06826] [INSPIRE].

[26] K. Ohmori, Worldsheet Geometries of Ambitwistor String, JHEP 06 (2015) 075 [arXiv: 1504.02675] [INSPIRE].

[27] E. Casali, Y. Geyer, L. Mason, R. Monteiro and K.A. Roehrig, New Ambitwistor String Theories, JHEP 11 (2015) 038 [arXiv:1506.08771] [INSPIRE].

[28] Y. Geyer, L. Mason, R. Monteiro and P. Tourkine, Loop Integrands for Scattering Amplitudes from the Riemann Sphere, Phys. Rev. Lett. 115 (2015) 121603 [arXiv:1507.00321] [INSPIRE].

[29] N.E.J. Bjerrum-Bohr, P.H. Damgaard, P. Tourkine and P. Vanhove, Scattering Equations and String Theory Amplitudes, Phys. Rev. D 90 (2014) 106002 [arXiv: 1403.4553] [InSPIRE].

[30] C. Baadsgaard, N.E.J. Bjerrum-Bohr, J.L. Bourjaily and P.H. Damgaard, Integration Rules for Scattering Equations, JHEP 09 (2015) 129 [arXiv:1506.06137] [INSPIRE].

[31] C. Baadsgaard, N.E.J. Bjerrum-Bohr, J.L. Bourjaily and P.H. Damgaard, Scattering Equations and Feynman Diagrams, JHEP 09 (2015) 136 [arXiv: 1507.00997] [INSPIRE].

[32] C. Baadsgaard, N.E.J. Bjerrum-Bohr, J.L. Bourjaily, P.H. Damgaard and B. Feng, Integration Rules for Loop Scattering Equations, JHEP 11 (2015) 080 [arXiv:1508.03627] [INSPIRE].

[33] L. Dolan and P. Goddard, The Polynomial Form of the Scattering Equations, JHEP 07 (2014) 029 [arXiv: 1402.7374] [INSPIRE].

[34] C. Kalousios, Scattering equations, generating functions and all massless five point tree amplitudes, JHEP 05 (2015) 054 [arXiv: 1502.07711] [INSPIRE].

[35] R. Huang, J. Rao, B. Feng and Y.-H. He, An Algebraic Approach to the Scattering Equations, JHEP 12 (2015) 056 [arXiv: 1509.04483] [INSPIRE].

[36] M. Sogaard and Y. Zhang, Scattering Equations and Global Duality of Residues, arXiv: 1509.08897 [INSPIRE].

[37] L. Dolan and P. Goddard, General Solution of the Scattering Equations, arXiv:1511.09441 [INSPIRE].

[38] C. Cardona and C. Kalousios, Elimination and recursions in the scattering equations, Phys. Lett. B 756 (2016) 180 [arXiv:1511.05915] [INSPIRE]. 
[39] C. Cardona and C. Kalousios, Comments on the evaluation of massless scattering, JHEP 01 (2016) 178 [arXiv: 1509.08908] [INSPIRE].

[40] F. Cachazo and H. Gomez, Computation of Contour Integrals on $\mathcal{M}_{0, n}$, JHEP 04 (2016) 108 [arXiv: 1505.03571] [INSPIRE].

[41] S. He and E.Y. Yuan, One-loop Scattering Equations and Amplitudes from Forward Limit, Phys. Rev. D 92 (2015) 105004 [arXiv: 1508.06027] [INSPIRE].

[42] Y. Geyer, L. Mason, R. Monteiro and P. Tourkine, One-loop amplitudes on the Riemann sphere, JHEP 03 (2016) 114 [arXiv:1511.06315] [INSPIRE].

[43] F. Cachazo, S. He and E.Y. Yuan, One-Loop Corrections from Higher Dimensional Tree Amplitudes, arXiv:1512.05001 [inSPIRE].

[44] C. Baadsgaard, N.E.J. Bjerrum-Bohr, J.L. Bourjaily, S. Caron-Huot, P.H. Damgaard and B. Feng, New Representations of the Perturbative S-matrix, Phys. Rev. Lett. 116 (2016) 061601 [arXiv:1509.02169] [INSPIRE].

[45] R. Huang, Q. Jin, J. Rao, K. Zhou and B. Feng, The Q-cut Representation of One-loop Integrands and Unitarity Cut Method, JHEP 03 (2016) 057 [arXiv:1512.02860] [INSPIRE].

[46] S.G. Naculich, Scattering equations and BCJ relations for gauge and gravitational amplitudes with massive scalar particles, JHEP 09 (2014) 029 [arXiv:1407.7836] [INSPIRE].

[47] S.G. Naculich, CHY representations for gauge theory and gravity amplitudes with up to three massive particles, JHEP 05 (2015) 050 [arXiv:1501.03500] [INSPIRE].

[48] S.G. Naculich, Amplitudes for massive vector and scalar bosons in spontaneously-broken gauge theory from the CHY representation, JHEP 09 (2015) 122 [arXiv:1506.06134] [INSPIRE].

[49] C.S. Lam and Y.-P. Yao, Off-Shell CHY Amplitudes, Nucl. Phys. B 907 (2016) 678 [arXiv: 1511.05050] [INSPIRE].

[50] S. Caron-Huot, Loops and trees, JHEP 05 (2011) 080 [arXiv: 1007.3224] [INSPIRE]. 